\title{
Visualizing synaptic plasticity in vivo by large-scale imaging of endogenous AMPA receptors
}

Austin R. Graves ${ }^{1,2,5}$, Richard H. Roth ${ }^{1,5}$, Han L. Tan ${ }^{1,5}$, Qianwen Zhu ${ }^{1,5}$, Alexei M. Bygrave , $^{1,5}$, Elena Lopez-Ortega ${ }^{1,5}$, Ingie Hong ${ }^{1}$, Alina C. Spiegel ${ }^{1,2}$, Richard C. Johnson $^{1}$, Joshua T. Vogelstein ${ }^{2,3,4}$, Daniel J. Tward ${ }^{2,3,4}$, Michael I. Miller ${ }^{2,3,4}$, Richard L. Huganir ${ }^{1,2^{*}}$

${ }^{1}$ Department of Neuroscience, Johns Hopkins University School of Medicine

${ }^{2}$ Kavli Neuroscience Discovery Institute

${ }^{3}$ Center for Imaging Science, Johns Hopkins University School of Engineering

${ }^{4}$ Department of Biomedical Engineering, Johns Hopkins University

${ }^{5}$ Equal contribution

*For correspondence: rhuganir@jhmi.edu

\section{Abstract}

Elucidating how synaptic molecules such as AMPA receptors mediate neuronal communication and tracking their dynamic expression during behavior is crucial to understand cognition and disease, but current technological barriers preclude large-scale exploration of molecular dynamics in vivo. We have developed a suite of innovative methodologies that break through these barriers: a new knockin mouse line with fluorescently tagged endogenous AMPA receptors, two-photon imaging of hundreds of thousands of labeled synapses in behaving mice, and computer-vision-based automatic synapse detection. Using these tools, we can longitudinally track how the strength of synapses changes during behavior. We used this approach to generate an unprecedentedly detailed spatiotemporal map of synaptic plasticity underlying sensory experience. More generally, these tools can be used as an optical probe capable of measuring functional synapse strength across entire brain areas during any behavioral paradigm, describing complex system-wide changes with molecular precision.

\section{Introduction}

Recent applications of genetically encoded calcium indicators and high-density silicon electrodes have revolutionized our understanding of the cellular and circuit basis of behavior; however, technological barriers preclude similar exploration of the molecular basis of these processes in vivo. To investigate the physiological function of complex molecular systems in vivo, we require techniques to visualize endogenous proteins. Modern proteomic and transcriptomic methods provide biologists with myriad candidate proteins, but in many cases, there are no tools available to effectively study these targets at the level of endogenous proteins in vivo. For example, we are far from having reliable antibodies for the entire proteome, and even when antibodies are available, there are concerns regarding their target specificity. Another approach is to fluorescently tag proteins to visualize their dynamic expression in living tissue. Combined with in vivo two-photon (2p) microscopy, this approach enables detailed investigation of the molecular mechanisms underlying complex physiological and pathological systems.

AMPA-type glutamate receptors (AMPARs) are crucial molecules to study to understand the function and dynamics of the nervous system. AMPARs mediate the majority of fast excitatory synaptic transmission in the mammalian brain and their regulation is regarded as a 
key mechanism underlying long-lasting changes in synaptic efficacy that give rise to learning and memory (Huganir and Nicoll, 2013; Malinow and Malenka, 2002). Long-term potentiation (LTP) is characterized by increased AMPAR trafficking to the postsynaptic membrane and associated spine enlargement, which together result in a long-lasting increase in synaptic efficacy; whereas long-term depression (LTD) is characterized by removal of postsynaptic AMPARs, resulting in attenuated synaptic transmission (Anggono and Huganir, 2012; Nicoll, 2017). Impaired regulation of synaptic plasticity is associated with human neurological and psychiatric disease (Berryer et al., 2013; Henley and Wilkinson, 2016; Volk et al., 2015). Despite this clear link between synaptic plasticity and learning, as well as a thorough understanding of molecular mechanisms regulating AMPAR trafficking, very little is known regarding how changes in plasticity are distributed among trillions of synapses throughout the brain. In contrast to advanced strategies to observe and manipulate neuronal activity - using genetically encoded calcium indicators (Dombeck et al., 2010; Lin and Schnitzer, 2016; Xu et al., 2012) or optogenetics (Fenno et al., 2011), respectively - there are currently no methods to physiologically measure postsynaptic strength in vivo on a brain-wide scale.

To overcome this barrier, we developed a new knockin mouse line wherein the AMPAR GluA1 subunit is tagged with super ecliptic pHluorin (SEP), a pH sensitive variant of GFP that fluoresces at neutral $\mathrm{pH}$ and is quenched at acidic $\mathrm{pH}$ (Miesenbock et al., 1998). When coupled to the extracellular N-terminal domain of the AMPAR, this SEP tag reports the concentration of functional receptors at the cell surface, as the fluorescence of receptors localized in acidic, internal compartments such as endosomes and Golgi is quenched. Our genetic labeling strategy also avoids confounds arising from manipulation of the AMPAR C-terminus, a region important for proper function and trafficking to the postsynaptic membrane (Sheng et al., 2018; Zhou et al., 2018). Many groups have used overexpression of SEP-tagged AMPARs in neuronal culture to study AMPAR trafficking in vitro (Araki et al., 2015; Ashby et al., 2004; Kopec et al., 2006; Makino and Malinow, 2009; Patterson et al., 2010; Roth et al., 2017). In addition, previous work using overexpression of SEP-tagged AMPARs in vivo has provided valuable insights regarding the molecular mechanisms of behaviorally relevant plasticity (Diering et al., 2017; El-Boustani et al., 2018; Makino and Malinow, 2009; Miyamoto et al., 2021; Roth et al., 2019; Suresh and Dunaevsky, 2017; Tan et al., 2020; Zhang et al., 2015), but these methods enable receptor visualization in only a sparse subset of cells and exogenous overexpression may result in protein mistargeting and dysregulation. The novel genetic labeling strategy presented here avoids these confounds, allowing visualization of endogenous AMPAR expression in a manner that does not impair synaptic function, plasticity, or behavior. Used in conjunction with in vivo $2 p$ microscopy, this novel SEP-GluA1 knockin mouse is the first tool that enables longitudinal tracking of synaptic plasticity underlying behavior at brain-wide scale with single-synapse resolution. Finally, we present a suite of algorithms to automatically detect and segment hundreds of thousands of fluorescently labeled AMPARs in vivo, enabling longitudinal tracking of synaptic plasticity across entire brain regions in awake behaving mice.

\section{Results}

\section{SEP-GluA1 knockin mouse line labels excitatory synapses}

Using homologous recombination, we generated a mouse knockin line that inserts SEP into the N-terminus of GluA1. Homozygous knockin mice are viable, breed well, and appear to be physiologically and behaviorally normal (see below). This approach fluorescently labels all GluA1-containing AMPARs in the mice (Fig. 1a-c), enabling robust visualization of excitatory synapses throughout the entire brain (Fig. 1 Supplement 1). We did observe a decrease in GluA1 
mRNA and protein expression in our homozygote knockin line compared to wild type (WT; Fig. $1 d-f)$, most likely due to decreased stability of the resulting mRNA. Using biochemical fractionation to isolate synapses from mouse hippocampal tissue, we observed reduced expression of GluA1 in the postsynaptic density (PSD, 56.6\% of WT mice) and in total membrane protein levels (P2, 44.4\% of WT mice; Fig. 1e-f). In contrast, we observed a trend of increased levels of GluA2 (P2, 107.9\% of WT mice; PSD, $115.8 \%$ of WT mice) and GluA3 (P2, 113.4\% of WT mice; PSD, $125.6 \%$ of WT mice) subunits in knockin mice, although these changes were not significant (Fig. 1e-f). These results suggest that there might be a small compensatory increase of GluA2/GluA3 in the knockin line as a result of decreased GluA1 expression. Western blots of total lysates from either whole brain or individual regions (hippocampus, cortex, and cerebellum) revealed that GluA1 expression levels were consistent across brain regions in knockin mice (Fig. 1 Supplement 2).
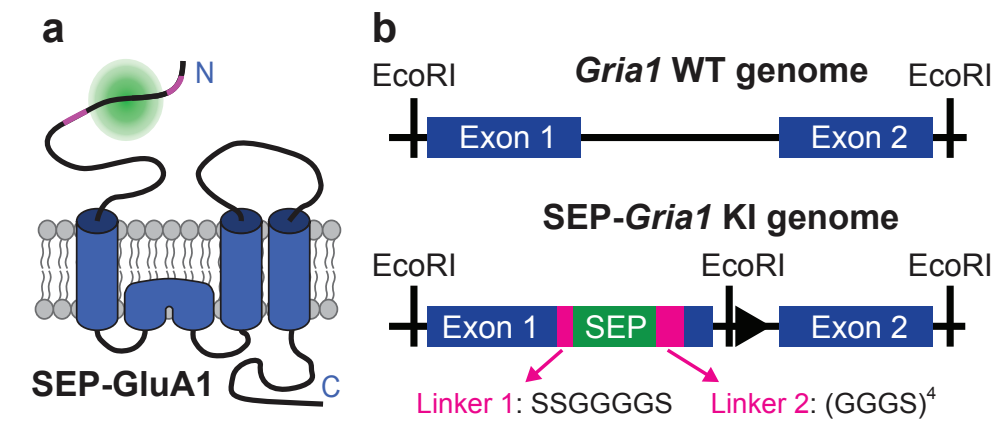

C Fluorescent image of WT and SEP-GluA1
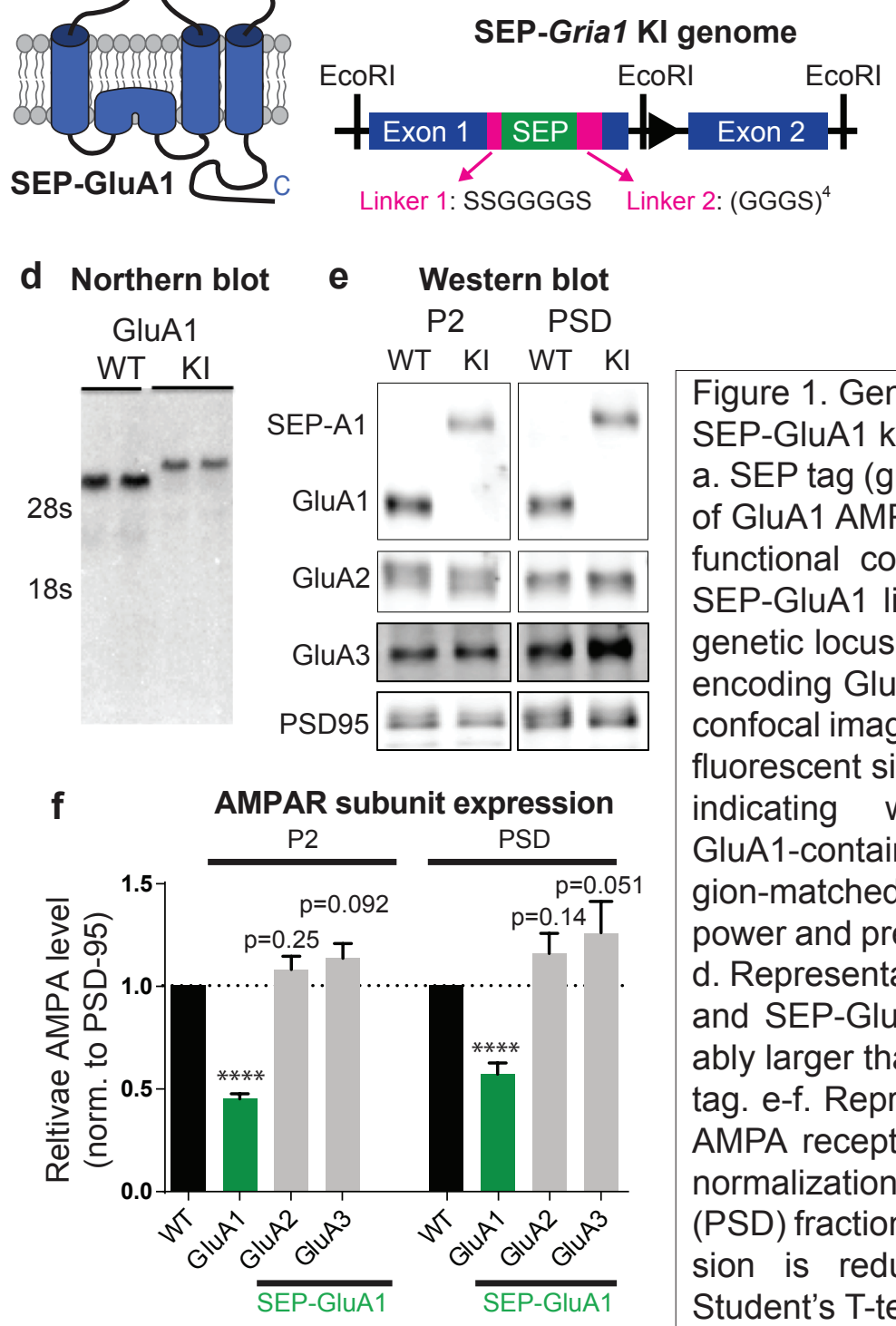

Figure 1. Generation and biochemical characterization of SEP-GluA1 knockin mouse line.

a. SEP tag (green) was targeted to extracellular N-terminus of GluA1 AMPARs (blue), enabling visualization of only the functional complement of AMPARs on the cell surface. SEP-GluA1 linkers depicted in magenta. b. Schematic of genetic locus of SEP tag on Exon 1, within the Gria1 gene encoding GluA1. Two linkers flank the SEP insert. c. Live, confocal image of acute slice of SEP-GluA1. Note the bright fluorescent signal throughout hippocampus and neocortex, indicating widespread expression of SEP-labeled GluA1-containing AMPARs. Age- and coronal-region-matched WT tissue was imaged with the same laser power and presented with identical contrast as SEP-GluA1. d. Representative Northern blot of mRNA expression of WT and SEP-GluA1 knockin (KI) mice. SEP-GluA1 is noticeably larger than WT GluA1, due to the inclusion of the SEP tag. e-f. Representative Western blot and quantification of AMPA receptor subunit expression in hippocampus after normalization to PSD95 in the P2 and postsynaptic density (PSD) fractions of WT and SEP-GluA1 mice. GluA1 expression is reduced relative to WT $\left(n=7 ;{ }^{* * *} p<0.0001\right.$, Student's T-test). 

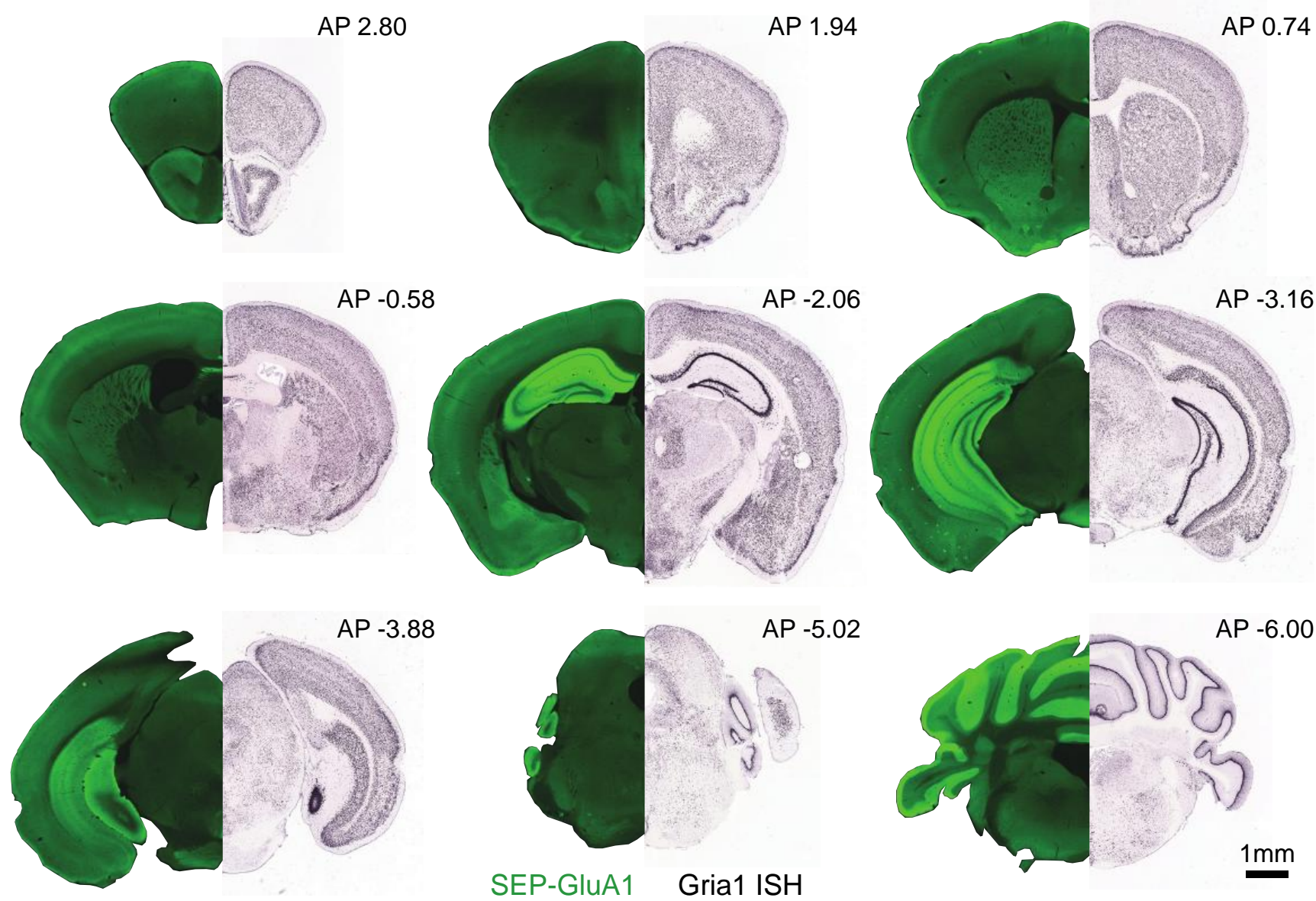

Figure 1 Supplement 1. Expression atlases of SEP-GluA1 and Gria1. Images are endogenous SEPGluA1 signal (left side, PFA-fixed sections imaged on Keyence slide scanner at 10x) and in situ hybridization of Gria1 mRNA expression (right side, from Allen Brain Atlas). Homozygous SEP-GluA1 brains (100 $\mu \mathrm{m}$ thick sections) at indicated anterior/posterior positions relative to bregma. SEP-GluA1 expression pattern is similar to GluA1 mRNA expression in WT mice throughout the brain.
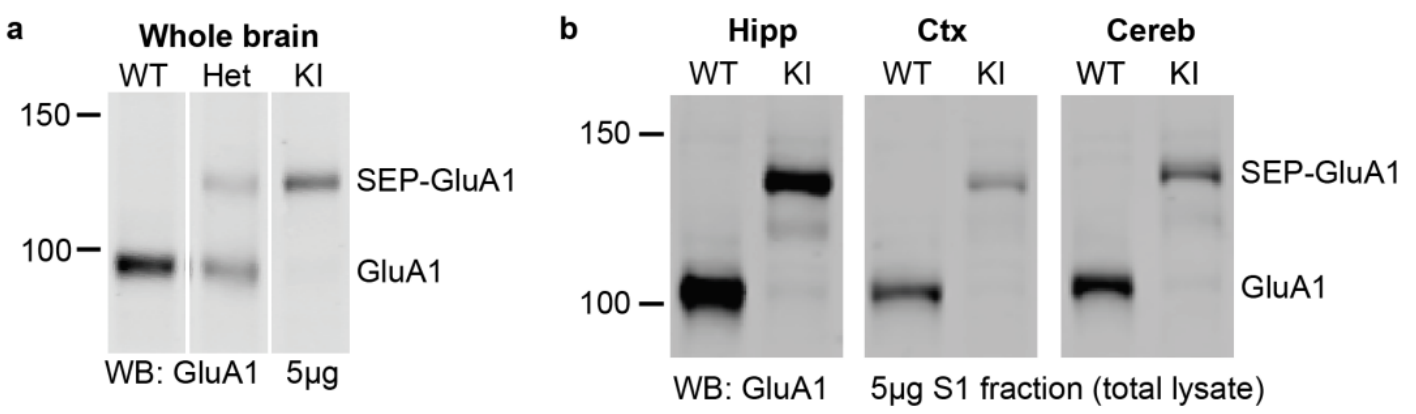

Figure 1 Supplement 2. Region specific differences in GluA1 expression in SEP-GluA1 KI mice. a. Whole brain total homogenate $(5 \mu \mathrm{g})$ from wildtype (WT), heterozygous SEP-GluA1 (Het) or homozygous SEP-GluA1 (KI) mice was blotted for GluA1. Samples run on the same gel, with image cropped to put lanes next to each other. b. S1 fractions $(5 \mu \mathrm{g})$, as a proxy for total homogenate, from hippocampus (Hipp), Cortex (Ctx) and Cerebellum (Cereb) were blotted for GluA1. In both (a) and (b) the higher band indicates SEP-GluA1 and shows reduced total levels of GluA1 in KI animals irrespective of brain region. 
a

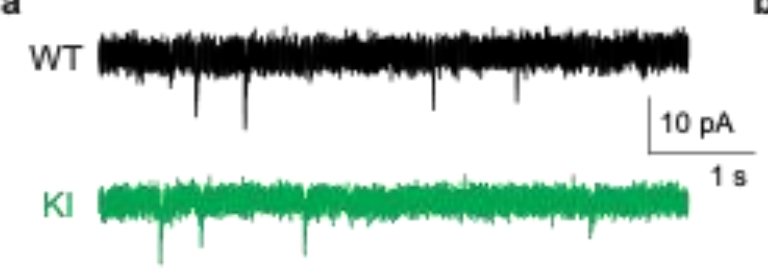

c

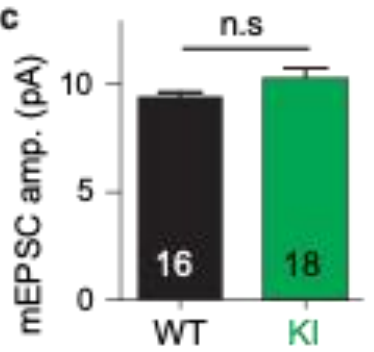

f

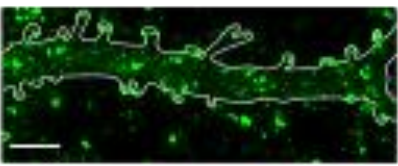

SEP-GluA1

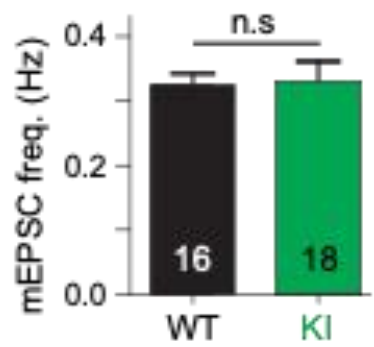

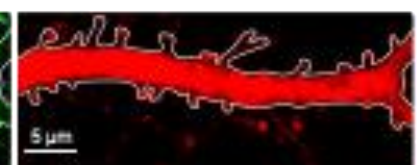

mCherry
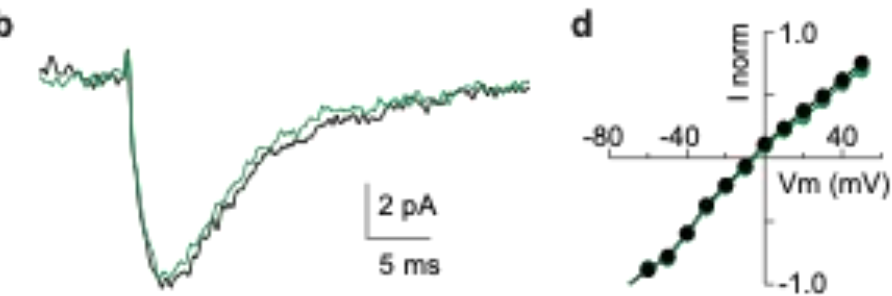
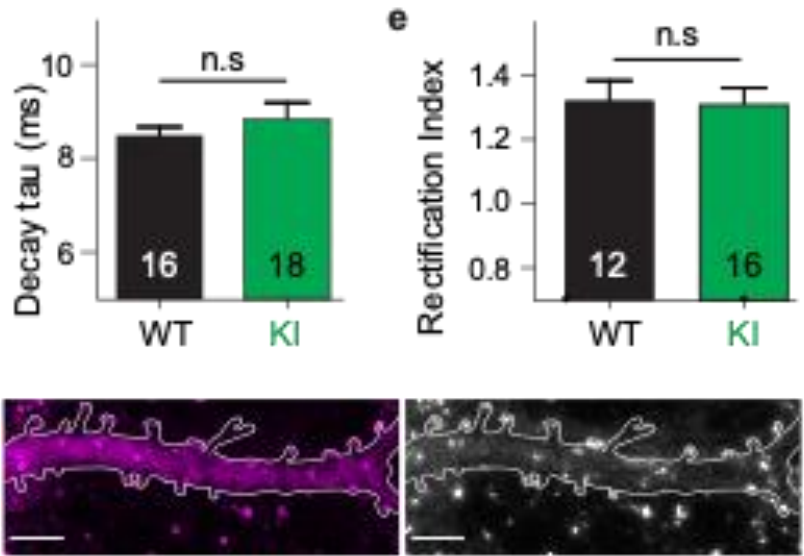

c-terminal-GluA1

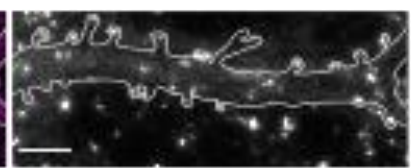

PSD-95 g

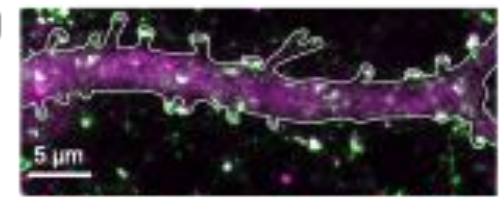

h

SEP-GluA1 + c-terminal GluA1

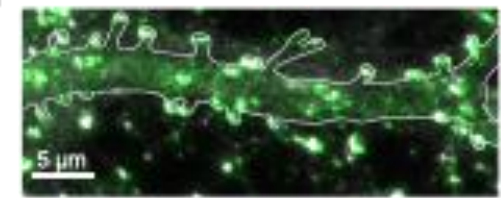

SEP-GluA1 + PSD-95

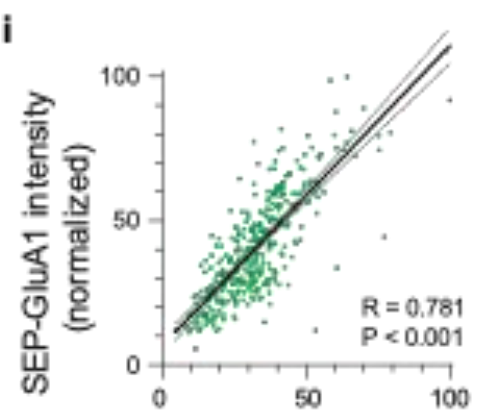

GluA1 antibody intensity (normalized)

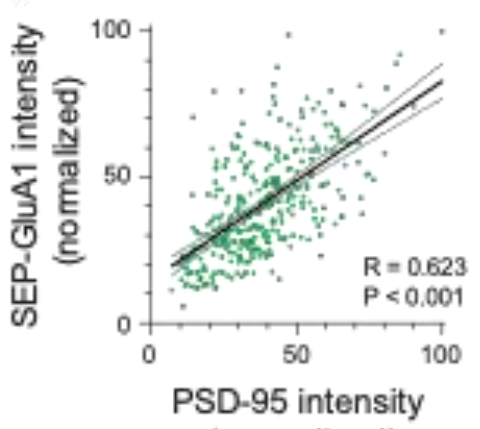

(normalized)

Figure 2. Normal synaptic physiology and receptor trafficking in SEP-GluA1 knockin mice. a-e. Wholecell voltage-clamp recordings from CA1 neurons in acute hippocampal slices of aged-matched wild type (WT) and homozygous SEP-GluA1 knockin (KI) littermates. a-b. Representative traces of miniature EPSCs from WT (black) and SEP-GluA1 knocking ("Kl”; green) mice. c. Quantification of amplitude, frequency, and kinetics of miniature EPSCs. No differences were observed in any electrophysiological parameters between WT and $\mathrm{KI}$ mice. $\mathrm{N}=16$ and 18 cells from WT and $\mathrm{KI}$, respectively. Unpaired Ttests were used for all comparisons. Mean \pm SEM. mEPSC amplitude: WT 9.43 $\pm 0.20, \mathrm{~N}=16 ; \mathrm{KI}$ $10.26 \pm 0.51, \mathrm{~N}=18 ; \mathrm{P}>0.05$. Frequency: WT $0.33 \pm 0.02 ; \mathrm{KI} 0.33 \pm 0.03122 ; \mathrm{P}>0.05$. Rise time: WT $2.46 \pm 0.040 ; \mathrm{KI} 2.49 \pm 0.07 ; \mathrm{P}>0.05$. Tau decay: WT 8.49 $\pm 0.18 ; \mathrm{KI} 8.83 \pm 0.36 ; \mathrm{P}>0.05$. d-e. No differences in rectification were observed between WT and KI mice. Rectification index is the negative slope of the IV curve (between -10 and $-60 \mathrm{mV}$ ) divided by the positive slope (between +10 and +50 $\mathrm{mV})$. Mean \pm SEM. WT: $1.32 \pm 0.048, \mathrm{~N}=12$; KI: $1.33 \pm 0.042, \mathrm{~N}=16 ; \mathrm{P}>0.05$. $\mathbf{f}-\mathbf{h}$. Confocal images of cultured SEP-GluA1 (green) neurons, with an mCherry cell-fill (red) and stained with antibodies for cterminal-GluA1 (magenta) and PSD-95 (gray). Overlap of SEP-GluA1 with c-terminal-GluA1 (G) and SEP-GluA1 with PSD-95 $(\mathrm{H})$ is rendered in white. I. Quantifying overlap between endogenous SEPGluA1 signal and immunofluorescence. A significant correlation is observed between the fluorescent intensity of endogenous SEP and the immunofluorescent signal of both GluA1 $(R=0.781, p<0.001$, Pearson correlation, $n=332$ spines) and PSD-95 ( $R=0.623, p<0.001$, Pearson correlation, $n=332$ spines). Lines represent linear regression (thick black) with 95\% confidence interval (thin gray). 
To validate the physiological function of our knockin line, we made whole-cell voltage-clamp recordings from CA1 pyramidal neurons in acute hippocampal slices of WT and homozygous SEP-GluA1 littermates. We observed no deficits in synaptic physiology or receptor trafficking in SEP-GluA1 mice (Fig. 2). Our electrophysiological data in particular support that synapses with fluorescently labeled AMPARs function identically to WT synapses, with no discernable differences in the amplitude, frequency, or kinetics of miniature excitatory postsynaptic currents (mini EPSCs; Fig. 2a-c) or rectification of the EPSCs (Fig. 2d-e). We also observed that SEPtagged GluA1 receptors were properly trafficked to the postsynaptic site and colocalized normally with other postsynaptic proteins, such as PSD-95 (Fig. 2f-h). There was a highly significant correlation between SEP-GluA1 signal and immunofluorescence intensity of both PSD-95 and c-terminal GluA1 antibodies, indicating that all GluA1 receptors express SEP in our knockin mouse line (Fig. 2g-i). In contrast, we observed a trend of increased levels of GluA2 (P2, 107.9\% of WT mice; PSD, $115.8 \%$ of WT mice) and GluA3 (P2, 113.4\% of WT mice; PSD, $125.6 \%$ of WT mice) subunits in knockin mice, although these changes were not significant (Fig. $1 \mathrm{e}-\mathrm{f})$. These results suggest that there might be a small compensatory increase of GluA2/GluA3 in the knockin line as a result of decreased GluA1 expression. Western blots of total lysates from either whole brain or individual regions (hippocampus, cortex, and cerebellum) revealed that GluA1 expression levels were consistent across brain regions in knockin mice (Fig. 1 Supplement 2).

\section{Intact synaptic plasticity and normal behavior in SEP-GluA1 knockin mice}

Homeostatic and Hebbian plasticity are the two major forms of synaptic plasticity that function cooperatively to keep neural circuits stable and plastic, respectively (Bliss and Lomo, 1973; O'Brien et al., 1998; Turrigiano et al., 1998). This novel knockin line represents a powerful tool to study both of these mechanisms, as they are each known to be expressed via dynamic regulation of synaptic AMPARs. To evaluate if homeostatic plasticity is intact in our knockin line, we made primary cultures of cortical neurons from homozygous SEP-GluA1 mice and WT littermates and treated them with either TTX or bicuculline for two days to induce up- or downscaling, respectively (Fig. 3a-b). As expected, WT neurons exhibited a significant reduction of surface AMPARs following bicuculline treatment and showed an elevation of surface GluA1 and GluA2 after TTX treatment. SEP-GluA1 knockin neurons displayed a similar bidirectional change of surface AMPARs following bicuculline and TTX treatments, indicating comparable homeostatic plasticity in SEP-GluA1 and WT mice. To assess Hebbian plasticity, we compared the expression of long-term potentiation (LTP) in WT and homozygous SEP-GluA1 littermates (Fig. 3c-e), as GluA1 knockout mice show deficits in LTP (Zamanillo et al., 1999). We performed whole-cell voltage-clamp recordings of synaptically evoked EPSCs in CA1 pyramidal cells in acute hippocampal slices of 3-4-week-old mice. After a baseline period of at least 5 minutes, a pairing stimulus consisting of $2-\mathrm{Hz}$ synaptic stimulation and somatic depolarization to $0 \mathrm{mV}$ was delivered, after which we resumed monitoring the amplitude of evoked EPSCs (Fig. 3d). This pairing protocol induced a long-lasting increase in EPSC amplitude in both WT and knockin neurons ( $n=8$ cells from each genotype), consistent with induction of LTP. We observed no differences in either induction or expression of LTP between WT and SEP-GluA1 littermates (Fig. 3e). Overall, these data strongly support that our knockin labeling strategy does not impair synaptic transmission and plasticity. 

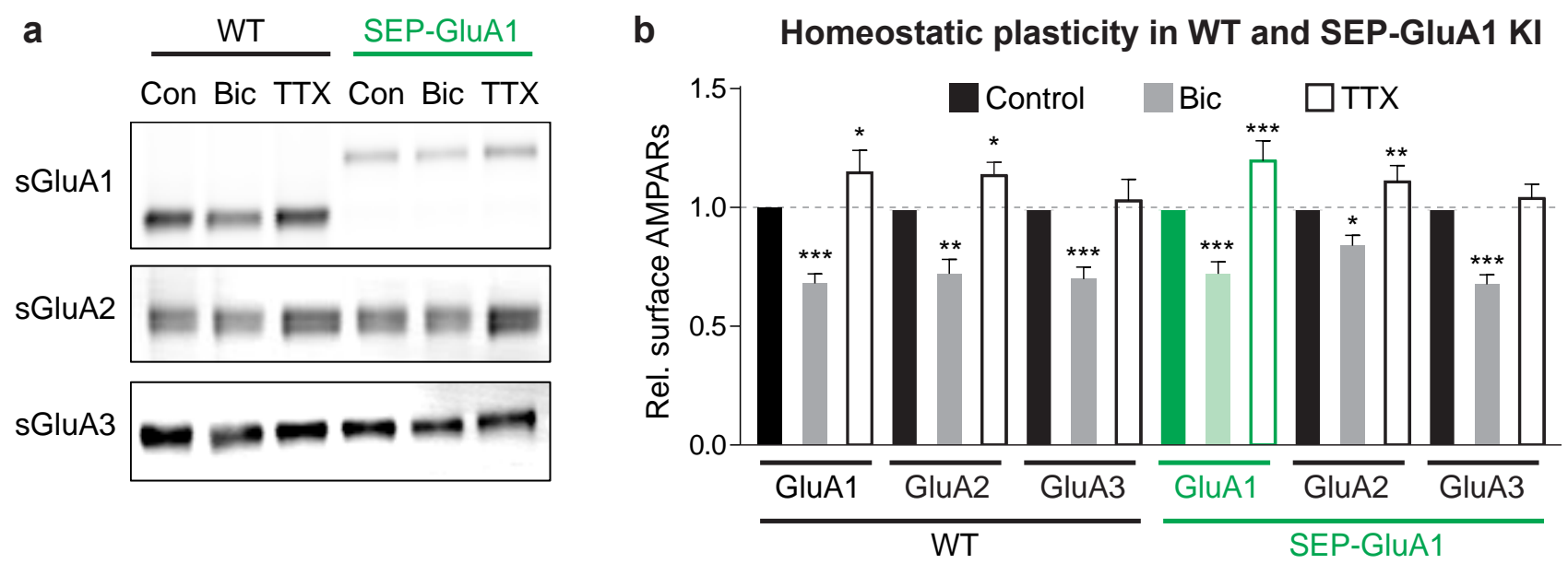

c Slice LTP setup

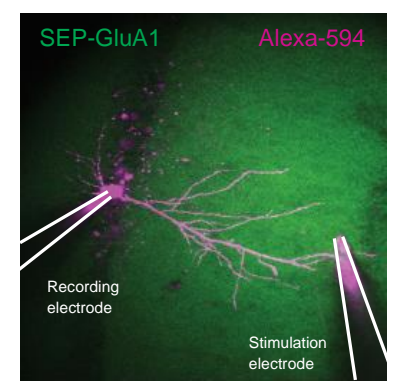

d

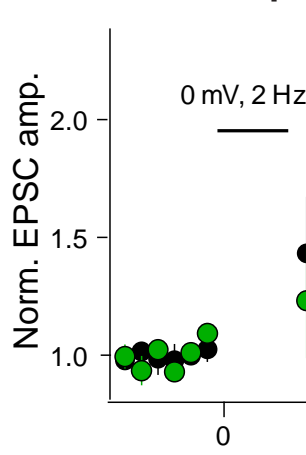

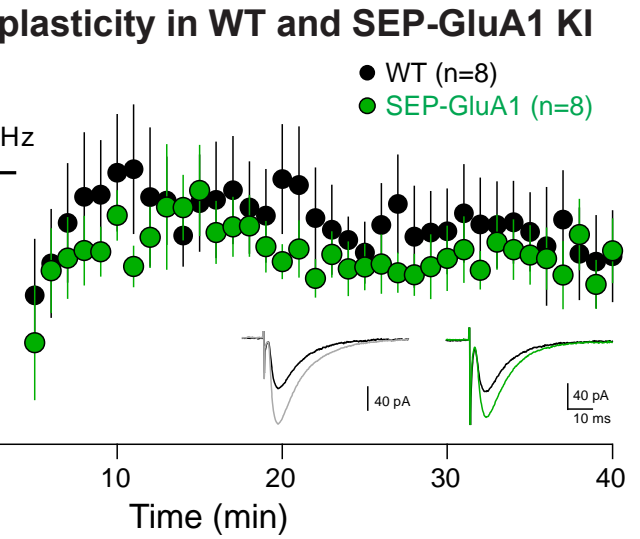

e

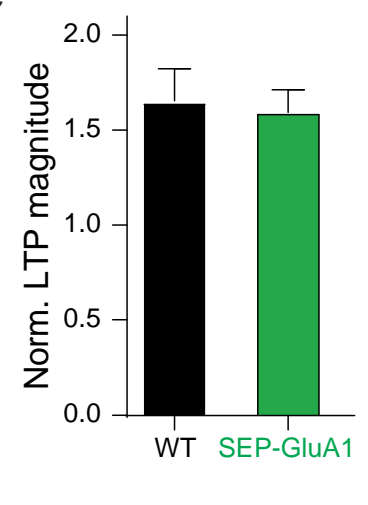

Figure 3. Normal homeostatic and Hebbian plasticity in SEP-GluA1 knockin mice. a. Representative Western blot of surface GluA1, GluA2, and GluA3 in WT and KI mouse neurons under baseline conditions (Con), following homeostatic downscaling in bicuculine (Bic), and following homeostatic upscaling in TTX. b. Bar plot of all homeostatic plasticity experiments $\left(n=7-8 ;{ }^{*} p<0.05\right.$, ${ }^{* *} p<0.01$, ${ }^{* * *} \mathrm{p}<0.001$; One-way ANOVA). c. Induction of long-term potentiation (LTP) in WT and SEP-GluA1 KI mice. Fluorescent image depicting experimental setup. CA1 pyramidal neurons were patched and filled with Alexa-594. A stimulating electrode in stratum radiatum was used to evoke EPSCs. After recording baseline EPSCs for at least 5 minutes, a pairing protocol consisting of 200 pulses was delivered at $2 \mathrm{~Hz}$. d. Average EPSC amplitude normalized to baseline for WT (black) and KI (green) littermates over course of LTP induction. Inset: Example traces of EPSCs from baseline (black) and 30-40 minutes following LTP induction (WT grey and KI green). e. Average change in EPSC amplitude normalized to the baseline period for WT and SEP-GluA1 KI littermates. A significant potentiation of EPSC amplitude was observed in both WT and KI mice, which was not different between genotypes, indicating normal induction and expression of LTP in SEP-GluA1 KI mice. Unpaired T-tests, $p>0.05$, mean \pm SEM; $W T: 1.66 \pm 0.17, n=8 ; K l: 1.59 \pm 0.34, n=8$. 
To further validate our SEP-GluA1 knockin line, we conducted a battery of behavioral experiments, as GluA1 knockout mice show deficits in several behaviors, including locomotor activity, anxiety, and spatial memory (Bannerman et al., 2004; Boerner et al., 2017; Bygrave et al., 2019; Sanderson et al., 2007). We assessed these behaviors in cohorts of SEP-GluA1 and WT littermates that were aged matched (both 6-10 weeks) and contained similar numbers of both sexes (SEP-GluA1: 9 females and 7 males; WT: 9 females and 9 males). We assessed locomotor activity by placing animals in an open arena and measuring the number of beam breaks during a 30-minute session. We observed no differences in the time course or total number of beam breaks between WT and SEP-GluA1 mice (Fig. 4a-b). Anxiety was assessed using an elevated plus maze, consisting of two closed arms and two open arms, suspended above the ground. We observed no differences in time spent in the open arms between WT and SEP-GluA1 mice (Fig. 4c-d). Spatial short-term memory was assessed using a $Y$ maze, consisting of three arms and surrounded by distal spatial cues. During the initial exposure phase, one arm was blocked with a clear plexiglass barrier. After exploring the two unblocked arms of the maze, mice were returned to their home cage for $1 \mathrm{~min}$, and then re-exposed to the maze for the test phase, wherein the barrier was removed. WT and SEP-GluA1 mice displayed a similar preference for the novel arm (Fig. 4e-f). Overall, these data strongly support that our knockin labeling strategy does not impair behavior, as SEP-GluA1 mice display comparable locomotion, anxiety, and short-term memory to WT animals.

\section{SEP-GluA1 reports synaptic plasticity in vitro}

To examine the function of individual SEP-GluA1 synapses, we used whole-cell voltageclamp recordings from primary cultures of homozygous SEP-GluA1 pyramidal neurons to measure evoked responses with $2 p$ glutamate uncaging. To visualize dendritic spines, neurons were filled with a red fluorescent dye via the patch pipette. Glutamate uncaging was targeted to the tip of spine heads (Fig. 5a) and the resulting uncaging-evoked excitatory postsynaptic current (uEPSC) was recorded (Fig. 5b). We found a significant correlation between SEP-GluA1 fluorescence intensity and UEPSC amplitude (Fig. 5c), indicating that SEP fluorescent intensity can be used as a proxy for synaptic strength.

We further used the SEP-GluA1 knockin line to track changes in synaptic strength following induction of synaptic plasticity in vitro in primary cultures of hippocampal neurons. Using $2 p$ imaging, glutamate uncaging, and whole-cell patch-clamp recordings from pyramidal neurons, we tracked SEP intensity and functional synaptic strength of spines that received high-frequency glutamate uncaging paired with postsynaptic depolarization versus spines that did not receive this pairing. We found that this pairing stimulus significantly increased UEPSC amplitude and SEP fluorescence in stimulated spines $(n=10 ; p<0.01$ relative to baseline, 1-way ANOVA), consistent with induction of LTP; whereas spines that received only postsynaptic depolarization unpaired with glutamate uncaging did not display similar changes ( $n=42$ spines; Fig. $5 d$-f). These data highlight the power of the SEP-GluA1 knockin line as a tool to monitor widespread synaptic strength and plasticity via fluorescence imaging. 
a

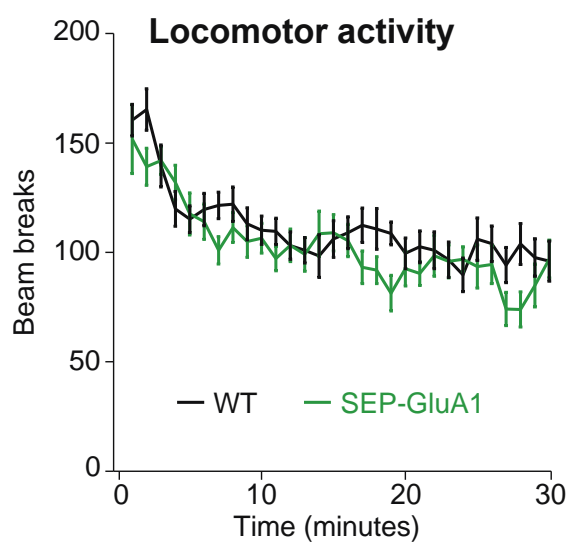

C

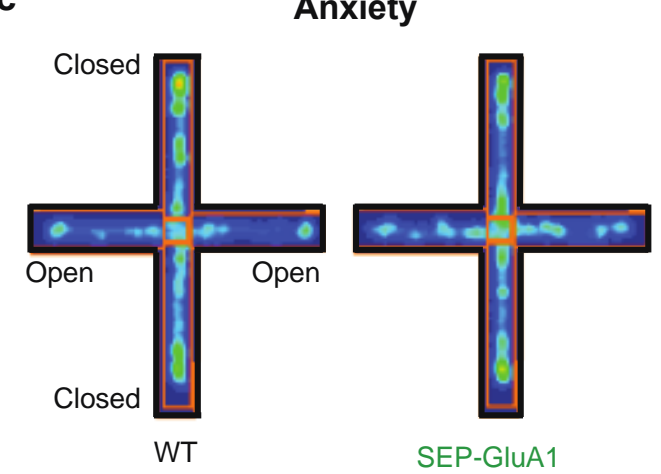

e Spatial novelty preference
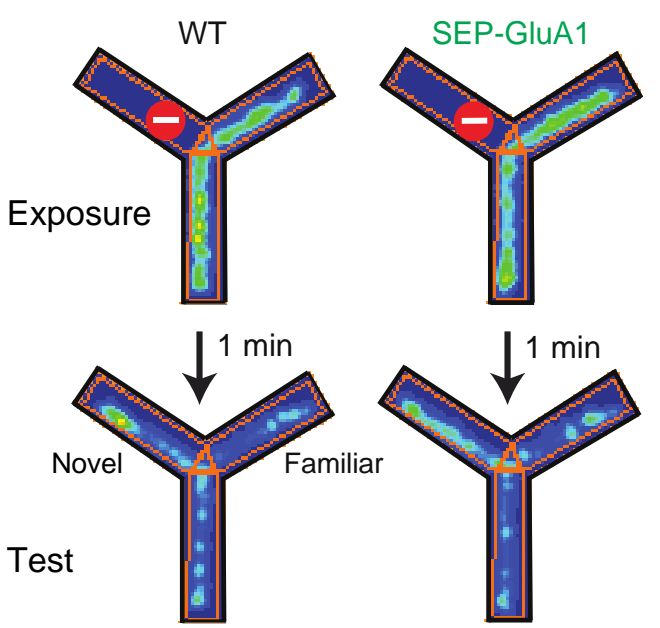
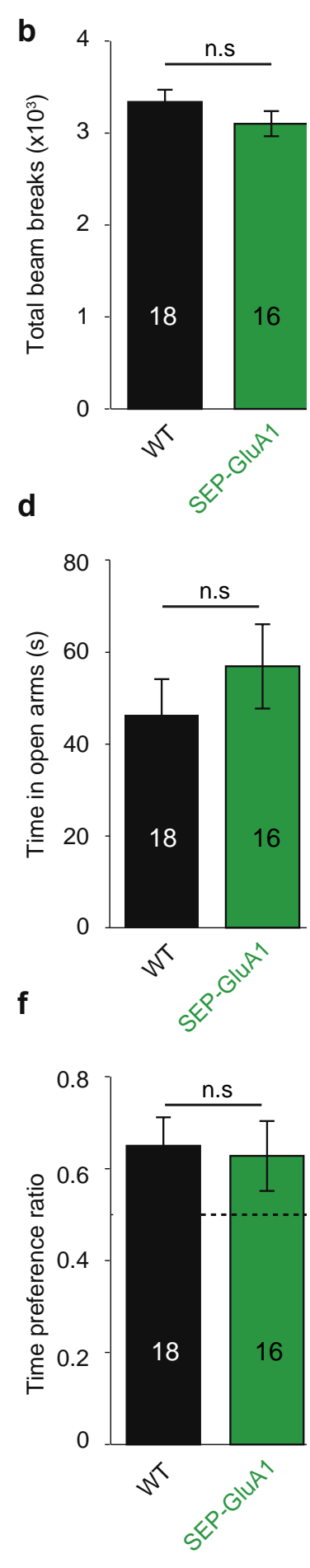

Figure 4. Normal behavior in SEPGluA1 knockin mice. a-b. SEP-GluA1 mice display normal locomotion. Agematched, WT $(n=18)$ and homozygous SEP-GluA1 KI $(n=16)$ littermates were placed in an open chamber and locomotion was assessed by counting the total number of beam breaks in a 30 minute session. No differences were detected between WT and KI mice (Repeated measures ANOVA; $\left.F_{1,30}=1.561, \quad P=0.221\right)$, between sexes $\left(F_{1,30}=1.346, P=0.255\right)$, or in a sex ${ }^{*}$ genotype dependent manner $\left(F_{1,30}=0.3, P=0.588\right)$. c-d. SEP-GluA1 mice display normal anxiety. WT $(n=18)$ and $K I(n=16)$ littermates were placed in an elevated plus maze and anxiety was assessed by measuring the time spent in the open arm. For representative WT and $\mathrm{KI}$ animals, time spent in a particular location is indicated in pseudo color, with warm colors indicating higher occupancy. No differences were detected between WT and $\mathrm{KI}$ mice (ANOVA; $\left.F_{1,30}=1.545, \quad P=0.224\right)$, between sexes $\left(F_{1,30}=0.160, P=0.692\right)$, or in a sex* genotype dependent manner $\left(F_{1,30}=4.139, \quad P=0.051\right)$. e-f. SEPGluA1 mice display normal short-term spatial memory. Spatial novelty preference was assessed in WT $(n=18)$ and $K I(n=16)$ littermates using a $\mathrm{Y}$-maze. WT and $\mathrm{KI}$ mice showed $\mathrm{a}$ preference for exploration of the novel arm. For representative WT and KI animals, time spent in a particular location is indicated in pseudo color, with warm colors indicating higher occupancy. There was no difference in the time preference ratio (time in novel arm/(time in novel arm + time in familiar arm)) between genotypes (ANOVA; $\quad F_{1,30}=0.004, \quad P=0.951$ ), between sexes $\left(F_{1,30}=1.277\right.$, $\mathrm{P}=0.267$ ), or in a sex*genotype dependent manner $\quad\left(F_{1,30}=2.434\right.$, $\mathrm{P}=0.129)$. Dotted line indicates chance level performance. 
a 2P glutamate uncaging on SEP-GluA1 spines
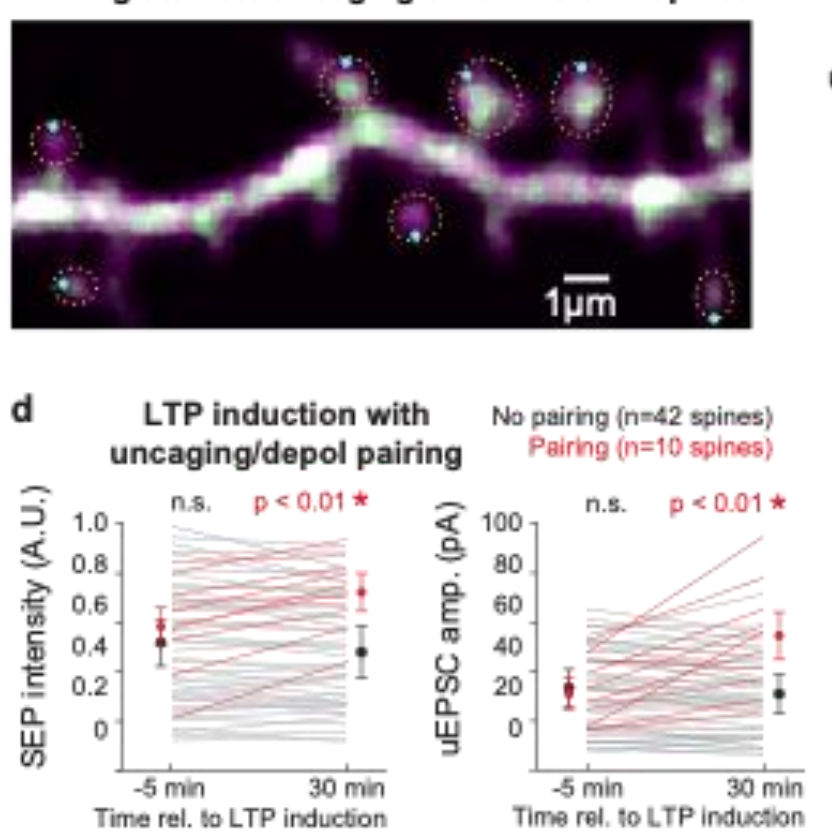

b Uncaging-evoked EPSC (UEPSC)

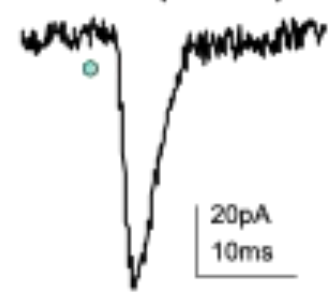

e

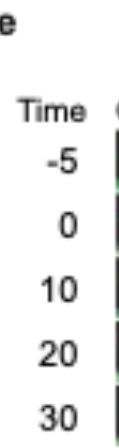

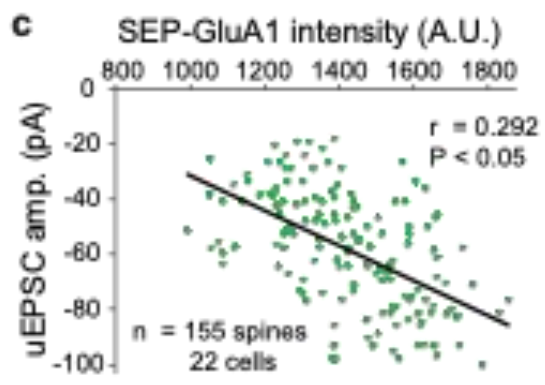

SEP-GluA1 expression

f Synaptic strength
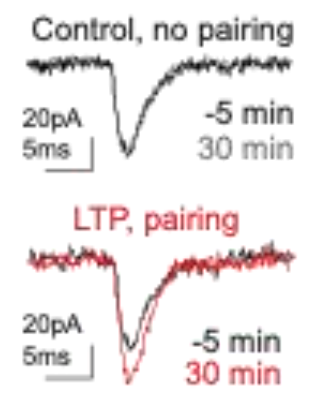

Figure 5. SEP intensity correlates with functional synaptic strength. a. $2 p$ image of a cultured SEPGluA1 neuron filled with Alexa 594 via somatic patch pipette, imaged at $910 \mathrm{~nm}$, with locations of glutamate uncaging indicated as blue dots and manually identified dendritic spines circled in yellow. b. Representative uncaging-evoked EPSC (uEPSC) following $1 \mathrm{~ms}$ pulse of $730 \mathrm{~nm}$ light at $20 \mathrm{~mW}$ (blue dot) in 2.5mM MNI-glutamate. c. Significant linear correlation between SEP-GluA1 intensity and uEPSC amplitude. SEP-GluA1 intensity was defined as the sum of green fluorescence intensity within manual synaptic annotations from 5 adjacent $0.5 \mu \mathrm{m}$-spaced z-planes. $\mathrm{n}=155$ spines from 22 cells $\left({ }^{*} p<0.05\right.$, Pearson's chi-squared test). d-f. Tracking synaptic plasticity with SEP-GluA1 in vitro. d. Plots of SEP-GluA1 intensity and UEPSC amplitude 5 minutes before and 30 minutes after delivery of an LTP induction stimulus consisting of high-frequency pairing of glutamate uncaging (30 pulses at $0.5 \mathrm{~Hz}, 1 \mathrm{~ms}$ pulse of $730 \mathrm{~nm}$ laser) and postsynaptic depolarization $(0 \mathrm{mV}$ for $0.5 \mathrm{sec}$, beginning concurrently with uncaging pulse). Red, spines that received LTP stimulus ( $n=10)$; black, spines that did not receive LTP stimulus $(n=42)$. ${ }^{*} p<0.01$ using one-way ANOVA. e. Longitudinal images of two control spines and one spine that received LTP stimulus. f. Representative uEPSCs during the baseline period ( $-5 \mathrm{~min})$ and 30 minutes after LTP induction. 


\section{SEP-GluA1 expression is regulated by a dynamic process in vivo}

To confirm that SEP-GluA1 fluorescence reports AMPAR dynamics in vivo, we used in vivo $2 p$ fluorescence recovery after photobleaching (FRAP) to measure SEP-GluA1 turnover rate. To visualize dendritic spines, AAV-CaMKII-Cre viral injections were performed in L2/3 of somatosensory cortex in SEP-GluA1 knockin x Ai9 (a tdTomato reporter line) double homozygous mice. The same area of cortex was imaged at baseline and following photobleaching (Fig. 6a). Spines targeted for photobleaching resulted in $\sim 50 \%$ reduction of fluorescence in SEP-GluA1 and $\sim 13 \%$ decrease in fluorescence in tdTomato (Fig. 6 Supplement 1). FRAP results confirmed that SEP-GluA1 signal in our knockin line represents slowly mobile molecules, supporting that SEP-AMPARs are normally targeted to the plasma membrane, in contrast to the tdTomato signal, which is freely diffusible and thus recovers more quickly (Fig. 6, Supplement 1). We also found that the SEP-GluA1 signal recovered in two phases after photobleaching (Fig. 6b). In the initial exponential phase (up to $30 \mathrm{~min}$ ), SEP-GluA1 reaches a plateau at $50 \%$ fluorescence recovery. This result suggests that about half of the GluA1containing AMPARs at the spines are part of the mobile fraction and, therefore, readily available to be exchanged with AMPAR pools outside the spine. This mobile fraction has been previously characterized by several in vitro and in vivo studies, although with different time scales. The timeline for mobile spine AMPAR exchange in cultured neurons has ranged from 5 minutes to periods longer than 15 minutes depending on the experimental conditions (Ashby et al., 2006; Fang et al., 2021; Frischknecht et al., 2009; Lee et al., 2017; Martin et al., 2009; Sharma et al., 2006). However, in the intact brain, the mobile AMPARs are exchanged after 30 minutes (Chen et al., 2021), in agreement with our observations. These results confirm that SEP-GluA1 is regulated by a cellular process that controls the dynamic exchange of molecules at the synapses, as would be expected for endogenous synaptic proteins.

The second phase at later time points represents a full recovery of SEP-GluA1 signal, indicating a complete turnover of synaptic GluA1-containing AMPARs in vivo within hours after photobleaching. This second AMPAR fraction is characterized by slower dynamics, probably due to protein interactions and molecular crowding within the postsynaptic density that limit AMPAR mobility (Bats et al., 2007; Li et al., 2016). The timescale presented in this study is supported by previous observations that establish a similar time course between synaptic AMPAR remodeling and AMPAR metabolic half-life ( $18 \mathrm{~h}$ for GluA1 subunit) in neuronal cultures (Mammen et al., 1997; O'Brien et al., 1998). In addition, these results demonstrate that we can track the same individual bleached and unbleached synapses longitudinally over several imaging sessions across consecutive days (Fig. $6 \mathrm{a}$ and $6 \mathrm{c}$ ). This is the first time that endogenous AMPAR recycling has been studied in vivo for up to $24 \mathrm{~h}$, providing valuable insights into basal AMPAR turnover dynamics in the intact brain and supporting that these tools can be used to longitudinally track synapse strength. Overall, these results confirm that SEP-GluA1-containing synapses are mobile and present similar dynamics as other in vitro and in vivo systems, supporting that our knockin labelling strategy does not perturb normal synaptic dynamics or function. 


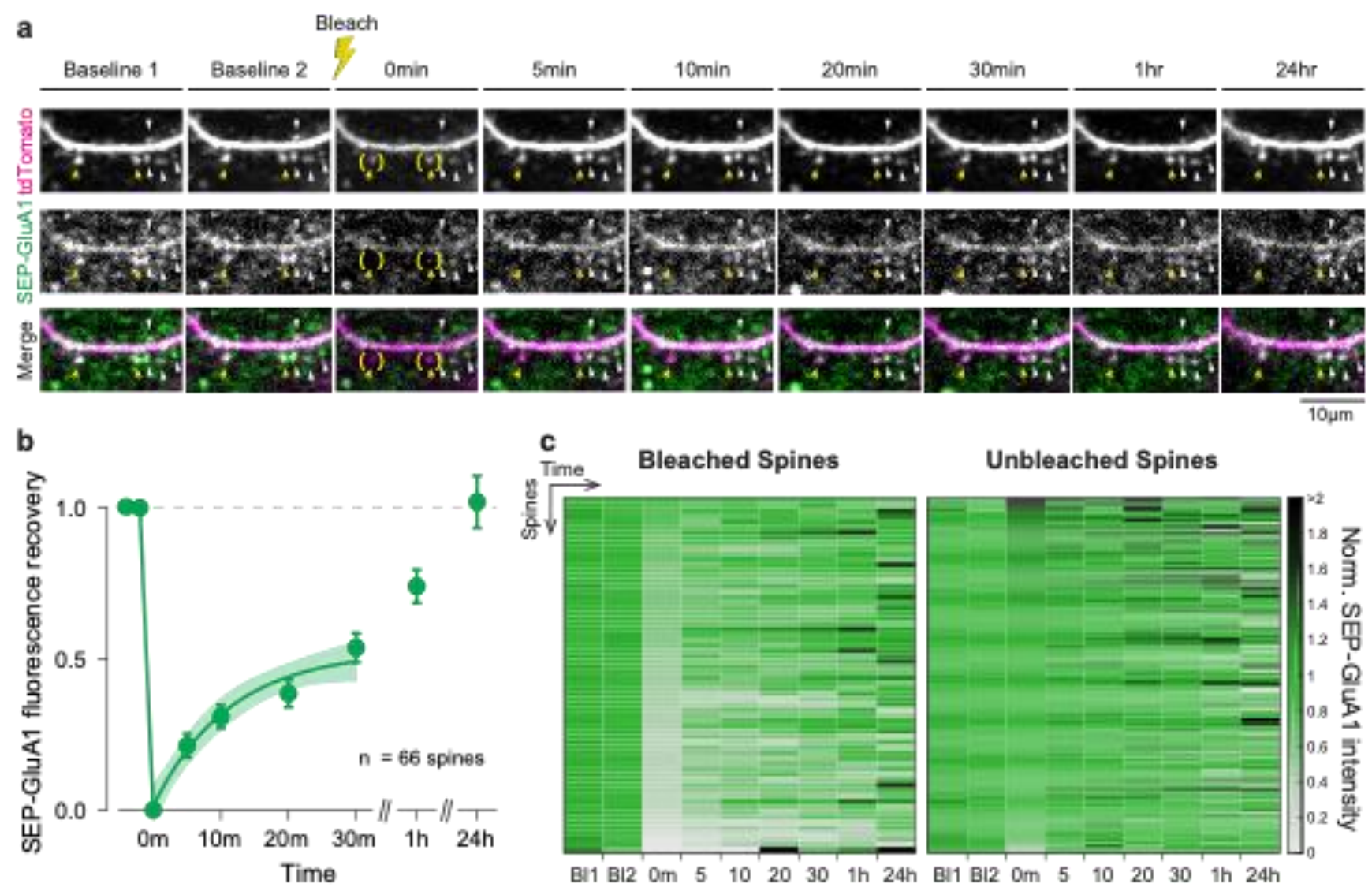

Figure 6. SEP-GluA1 signal completely recovers after photobleaching in vivo.

a. Representative in vivo $2 p$ images throughout fluorescence recovery after photobleaching (FRAP) in a SEP-GluA1 mouse. A sparse subset of neurons was filled with tdTomato to visualize dendrites and spines. Yellow arrows denote spines that were bleached (at $t=0 \mathrm{~min}$; bleaching area depicted in parentheses) and spines that were not bleached are indicated by white arrows. Scale bar 10 $\mathrm{mm}$. b. Fluorescence recovery of SEP-GluA1 signal after photobleaching in spines of L2/3 excitatory neurons in mouse somatosensory cortex. Symbols represent mean and error bars represent SEM. Time points between $0-30 \mathrm{~min}$ were fitted to a one-phase decay exponential curve (solid line), with plateau = $0.526+/-0.057$, rate constant of recovery $(\mathrm{k})=0.09+/-0.027$ (value $+/-$ SEM) and tau $=11.15 \mathrm{~min}$. Shaded area represents $95 \%$ confidence interval of the fit. $n=66$ spines from 3 mice. c. Heatmap of SEP-GluA1 signal from individual bleached and unbleached spines normalized to their respective baseline at different time points throughout FRAP. Rows represent individual spines sorted by signal intensity immediately after photobleaching $(t=0 \mathrm{~min})$. Bleached spines: $n=66$ spines from 3 mice. Unbleached spines: $\mathrm{n}=132$ spines from 3 mice. 


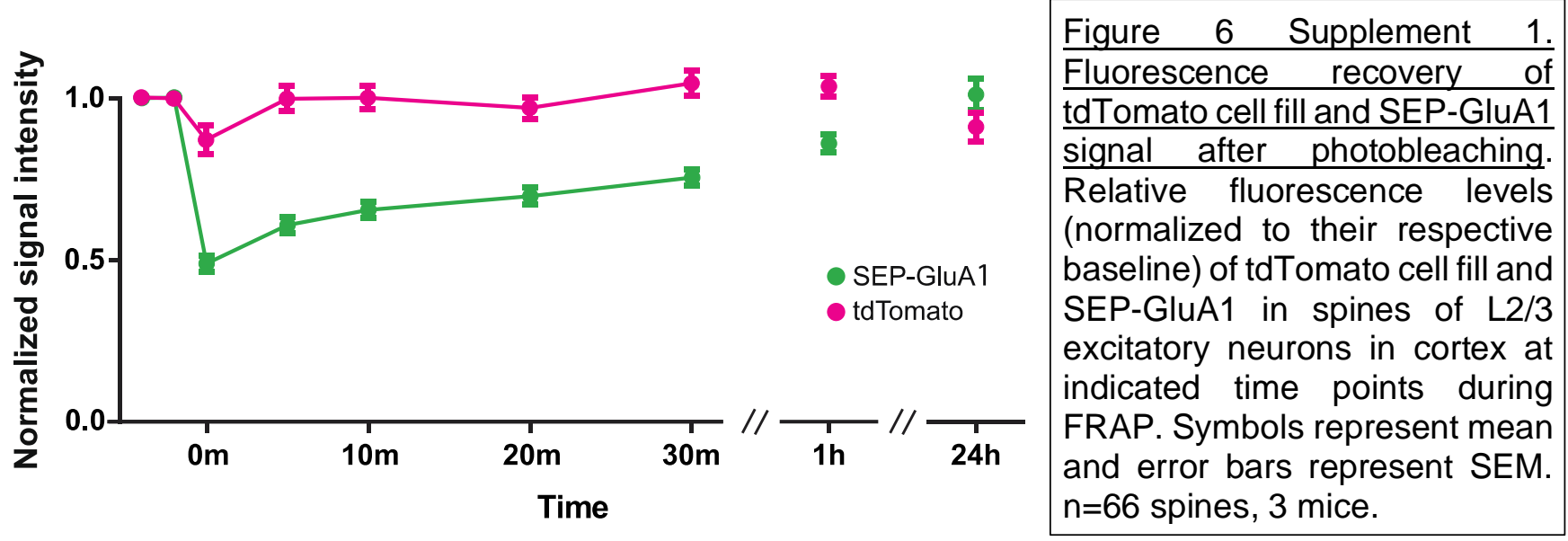

\section{Developing computational tools to detect and track labeled synapses in vivo}

To observe AMPAR dynamics in living mice on a large scale, we implanted cranial windows over somatosensory cortex in homozygous SEP-GluA1 mice and used $2 p$ microscopy to visualize endogenously labeled synapses (Fig. 7a-f and Movies 1-3). The observed bright green punctate fluorescence reflects synaptic enrichment of GluA1, likely corresponding to the functional complement of GluA1-containing AMPARs at the PSD (Fig. 7b,c). Given the richness and scale afforded by this knockin line (Movies 1-3), which endogenously labels all GluA1containing synapses throughout the brain, manual annotation of labeled synapses was not feasible. Thus, to automatically detect and segment extremely large numbers of SEP-labeled synapses, we developed an unsupervised machine learning algorithm based on 3D Wiener filtering, employing pre-whitened matched templates based on the mean appearance of manually annotated synapses relative to background noise. This approach enabled flexibility to tune segmentations based on accuracy criteria, such as tradeoffs between sensitivity and specificity, as well as prior information about synapse size and shape.

To validate our computer-vision-based automatic synapse detection platform, two expert synaptic anatomists manually annotated thousands of individual SEP-GluA1 synapses from in vivo volumes of somatosensory cortex (Fig. $7 \mathrm{f}$ ). We found relatively low inter-rater reliability ( $72.3 \%$ agreement, defined as $>50 \%$ shared voxels; Fig. 7 Supplement 1 ). Thus, rather than attempting to design an algorithm that reproduces highly variable human intuition, we chose to carefully define what an observed synapse looks like through a system of rules. Our segmentation algorithm is unique in that we interpret its output as the physical definition of a synapse. Our algorithm uses the following rules to define a synapse and its boundaries: 1) a candidate synapse is defined as a local maximum in an image blurred using a Gaussian kernel with standard deviation of 5 pixels in the xy plane and 1 pixel out of plane; 2 ) candidate synapses are not less than 3 pixels away from each other, as determined by a furthest first traversal; 3 ) synapses are ellipses in the xy plane, with eccentricity between 1.0-2.5; 4) synapses have an area between 20 and 150 pixels in the xy plane, corresponding to a circle of area $0.125-1.25$ $\mu \mathrm{m}^{2}$ (though ovals were also considered); these constraints were based on mean synapse size from electron microscopy datasets (Santuy et al., 2020); 5) a synapse shape is chosen to be the size and orientation and eccentricity that maximizes signal-to-noise ratio (SNR) (template matching); 6) SNR should be larger than the 90th percentile of 300 randomly chosen locations; 7) averaging 2 neighboring slices should increase SNR; 8) averaging 6 neighboring slices should decrease SNR. 
a

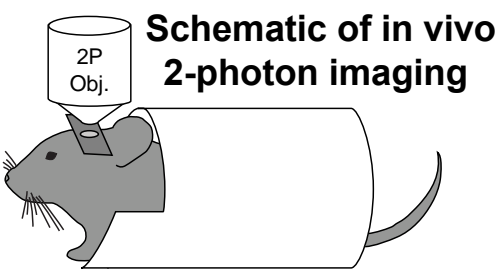

b Single in vivo imaging plane

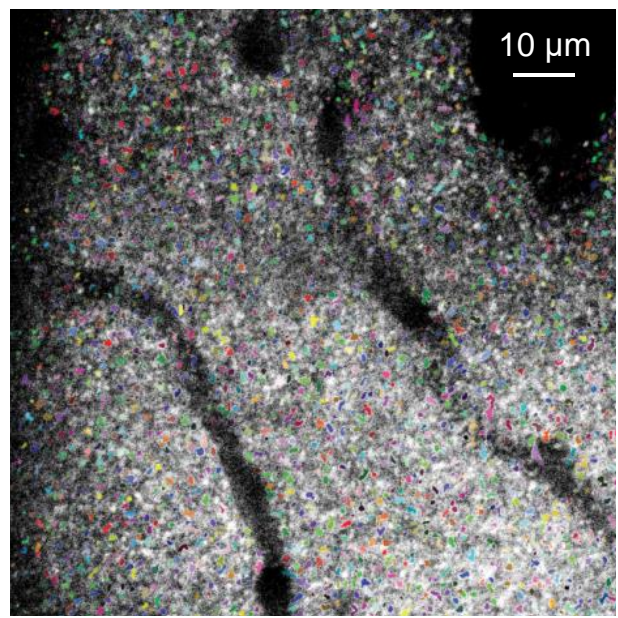

C Spine enrichment of SEP-GluA1

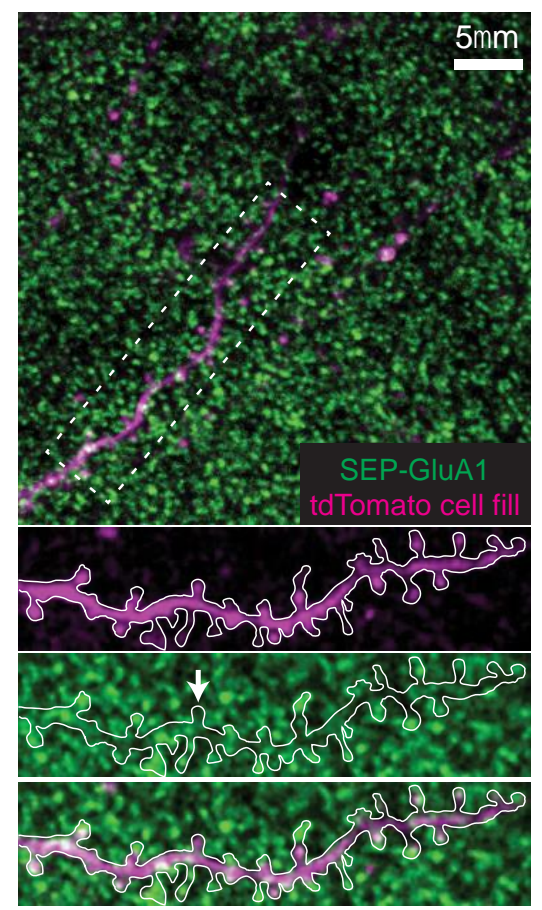

d Spine localization
of SEP-GluA1

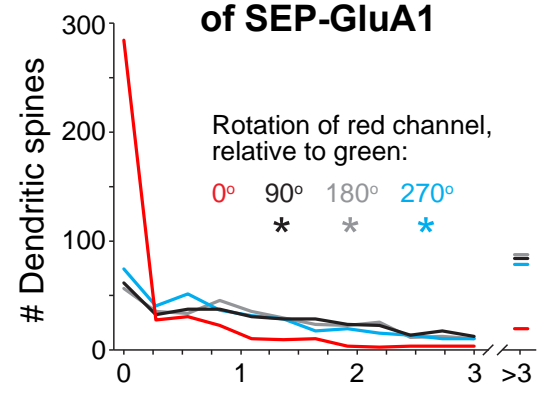

Dist. to nearest SEP-puncta $(\mathrm{m})$

\section{e Synapses are uniformly} detected throughout L1

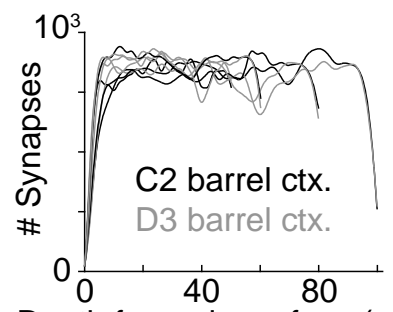

Depth from pia surface $(\mathrm{m})$ f

Visualization of SEP-GluA1containing synapses in vivo

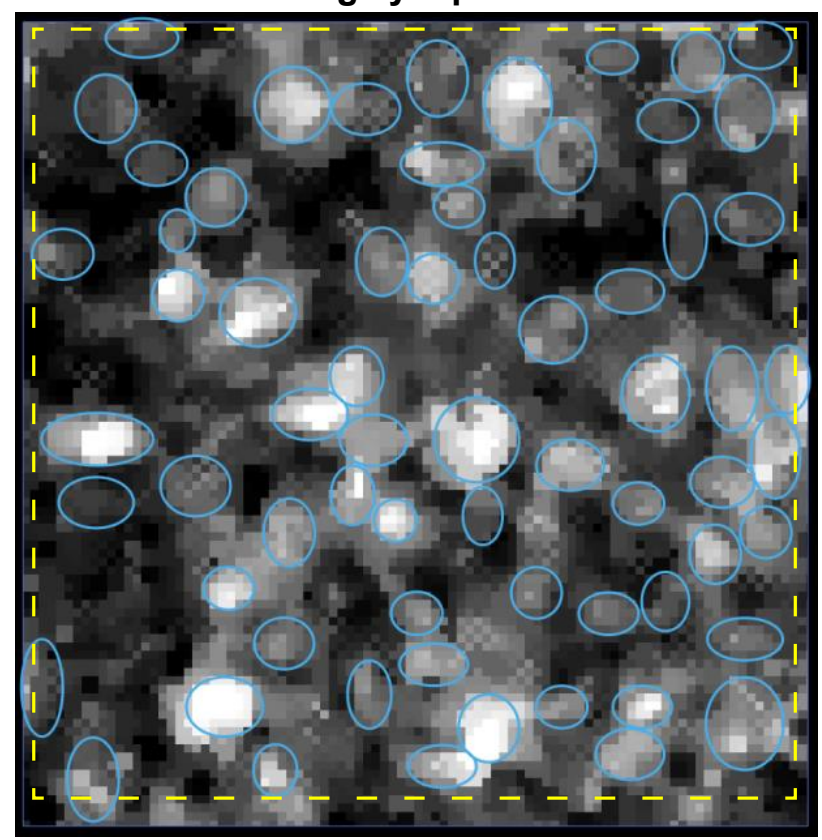

Manually annotated synapse
$1 \mathrm{~m}$
Automatic synapse detection and segmentation algorithm

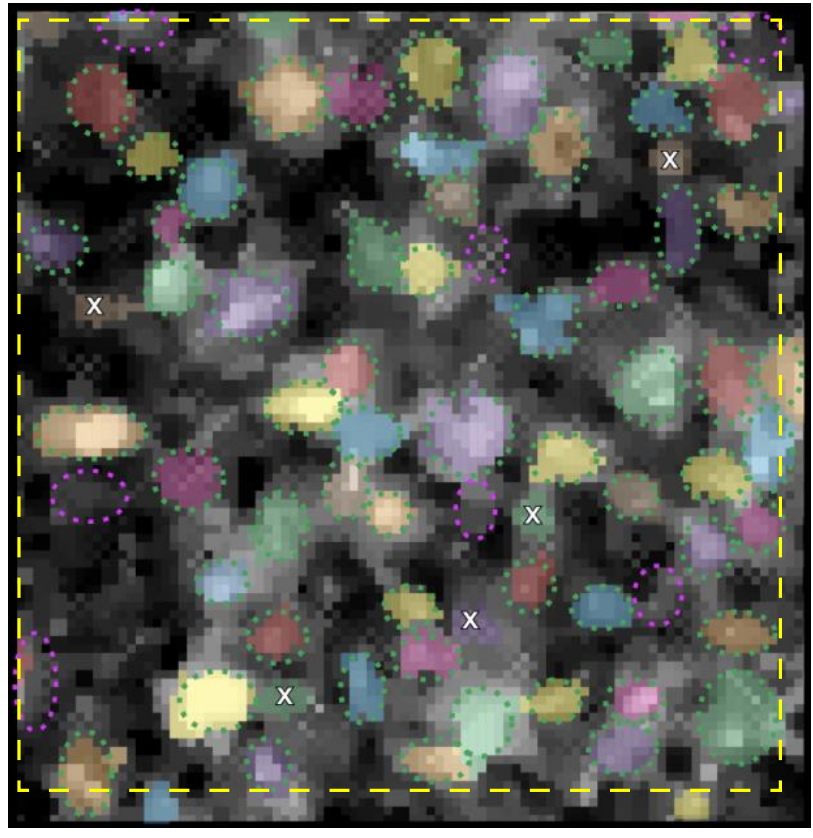

$\because$ Positive synapse asignment

is False-positive synapse asignment

$\because$ : False-negative synapse asignment 
Figure 7. Visualizing SEP-GluA1 synapses in vivo using $2 p$ microscopy. a. Schematic of in vivo $2 p$ imaging. b. Large-scale automatic detection and segmentation of SEP-GluA1-containing synapses in L1 barrel cortex. Automatically detected synapses are rendered in arbitrary colors. Dark areas likely correspond to either vasculature or cell bodies. c. Single in vivo imaging plane showing SEP-GluA1 synapses (green) and a single layer $2 / 3$ pyramidal cell filled with tdTomato (magenta). White arrow denotes a spine devoid of SEP-GluA1 signal. d. SEP-GluA1 is enriched in dendritic spines. $78 \%$ of automatically detected dendritic spines (visualized using a sparse tdTomato cell fill) contained a SEP GluA1 synapse, defined as edge-to-edge separation of red spines and green puncta $<0.25 \mu \mathrm{m}$. This overlap occurred at a substantially higher rate than chance, as the distance between spines (magenta channel) and their nearest SEP neighbor (green channel) significantly increased when the magenta channel was rotated either 90,180 , or $270^{\circ}$ relative to green $\left(n=504\right.$ spines; ${ }^{*} p<0.001$; Mann-Whitney $\mathrm{U}$ test, relative to unrotated). e. GluA1-containing synapses were uniformly detected throughout L1 barrel cortex, up to a depth of $100 \mu \mathrm{m}$ below the pia surface. $\mathbf{f}$. Left, single in vivo imaging plane displaying raw, unprocessed SEP-GluA1 signal, taken $47 \mu \mathrm{m}$ deep in layer 1 (L1) of barrel cortex. Putative GluA1-containing synapses are identified as bright puncta. Manual synaptic annotations are overlaid as blue ovals. Right, same cortical plane, but with automatically identified and segmented synapses rendered in arbitrary colors. Manual annotations are overlaid, recolored either green or magenta, corresponding to true positives (defined as $>50 \%$ of total 3D voxels shared between manual and automatic annotations) or false negative (defined as manual annotations that did not overlap with an automatic detection), respectively; false positives (defined as automatically detected synapses that did not overlap with a manual annotation) are indicated by an X. Scale bar is $1 \mu \mathrm{m}$.

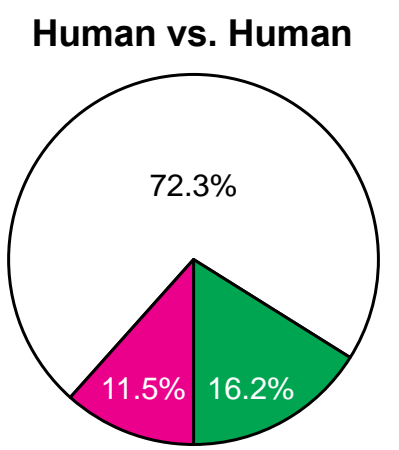

+Human1 +Human2 (positive agreement) +Human1 -Human2 (negative agreement) -Human1 +Human2 (negative agreement)
IHC Ground Truth

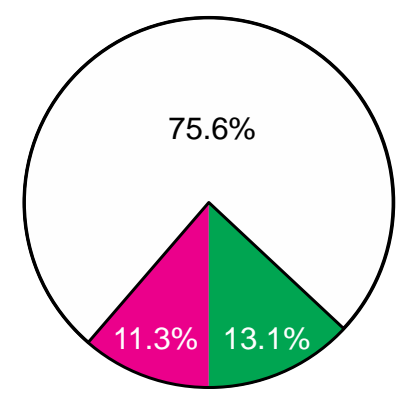

+SEP + Homer (true positive)

+ SEP -Homer (false positive)

-SEP + Homer (false negative)

\section{Human vs. Automatic}

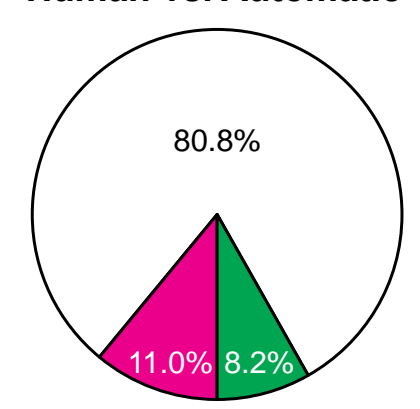

+Human +Automatic (positive agreement)

+Human -Automatic (false positive)

-Human +Automatic (false negative)

Figure 7 Supplement 1. Rates of agreement and error for synapse detection methods. For all plots, rates of agreement (positive annotation in both channels being compared) are shown in white and errors (positive annotation in one channel and negative in the other) are shown in magenta or green. Left, IHC Ground Truth is comparison of overlap of automatically detected SEP-GluA1 and Homer puncta in vitro. Middle, Human vs. Automatic is comparison of overlap of automatically detected SEPGluA1 and manually annotated SEP-GluA1 from the same volume of tissue imaged in vivo. Right, Human vs. Human is comparison of overlap between two different expert human annotators from the same volume of tissue imaged in vivo. For all comparisons, a threshold of $>50 \%$ shared voxels defined overlap. 
By filtering based on size in both the $X Y$ and $Z$ planes and by applying template matching, we were able to minimize false-positive detections, likely corresponding to either acquisition noise or extrasynaptic SEP-GluA1 receptors along the dendritic shaft. While fluorescent signal from extrasynaptic receptors is certainly present in our images (see Movies 1-3), our computational approach was largely successful in filtering out this smaller, more diffuse, and less punctate signal from our automated synapse detection and subsequent analyses.

To quantitatively assess ground truth for synapse detection, we performed immunohistochemical labeling of Homer, an abundant postsynaptic density protein, comparing rates of overlap between these two independent synaptic markers (Fig. 7 Supplement 2). As we currently do not have tools to label and visualize Homer expression in vivo, we imaged slices of barrel cortex from SEP-GluA1 mice, using the same $2 p$ beam path and identical acquisition settings as for in vivo experiments. SEP-GluA1 and Homer puncta were automatically detected in vitro using the same algorithm, with $50 \%$ of shared voxels defined as overlap. We found a true positive rate of $75.6 \%$ (overlap of SEP-GluA1 and Homer), a $13.1 \%$ false positive rate (SEP detected without Homer overlap), and an $11.3 \%$ false negative rate (Homer detected without SEP overlap; Fig. 7 Supplement 2e). This false negative rate is likely an overestimate of error, as this fraction could correspond to Homer-containing synapses that either don't contain detectable levels of the GluA1 subunit (i.e. synapses where GluA2/3 heterodimers predominate) or are so-called silent synapses that don't contain any AMPARs at all.

To further validate our synapse algorithm, we examined overlap between automatically detected synapses and dendritic spines using a sparse cell fill. Enrichment of SEP-GluA1 in spine heads was readily apparent (Fig. 7c), as 78\% of dendritic spines (visualized using a sparse tdTomato cell fill) contained a SEP-GluA1 synapse, defined as edge-to-edge separation of red spines and green puncta $<0.25 \mu \mathrm{m}$ (Fig. $7 \mathrm{c}-\mathrm{d}$ ). This overlap occurred at a substantially higher rate than chance, as the distance between spines (magenta channel) and their nearest SEPGluA1 neighbor (green channel) significantly increased when the magenta channel was rotated either 90,180 , or $270^{\circ}$ relative to green (Fig. $7 d$ ). SEP-GluA1 synapses were detected uniformly across depth within layer 1 (L1) of barrel cortex, up to $100 \mu \mathrm{m}$ below the pia surface (Fig. 7e).

Our automatic synapse detection algorithm enables robust identification and segmentation of hundreds of thousands of SEP-GluA1 synapses in vivo, with accuracy at least comparable to expert human annotators, but with vastly increase speed and scale. Indeed, we observed similar accuracy and error rates between human annotated and automatically detected synapses in the same cortical volumes. Using the same threshold for defining overlap of $50 \%$ shared voxels (see Fig. 7 Supplement 1), we found $80.8 \%$ agreement between automatic and human annotated synapses (green dashed ovals in Fig. 7f), with an $8.2 \%$ false positive rate (automatic detection without an overlapping human annotation; white $X$ in Fig. $7 f$ ) and an $11.0 \%$ rate of false negative (human annotation without overlapping automatic detection, magenta dashed ovals in Fig. $7 f$ and Fig. 7 Supplement 1). These accuracy rates are slightly better than the rate of agreement between two expert humans annotating the same volume (72.3\% agreement).

To accurately estimate our synaptic detection resolution boundaries, we measured the point-spread function (PSF) of our $2 p$ microscope (Fig. 7 Supplement 3). The resolution of our $2 p$ microscope in the $X$ and $Y$ directions is 0.55 and $0.57 \mu \mathrm{m}$, respectively (FWHM of reconstructed PSF), compared to $2.50 \mu \mathrm{m}$ in Z, suggesting that our ability to accurately segment boundaries of individual synapses in $X Y$ is greater compared to segmentation in Z. Given this relatively lower axial resolution inherent to $2 p$ microscopy, we are unable to accurately segment two closely abutting synapses that overlap within 3 adjacent $z$-sections (each separated by $1 \mu \mathrm{m})$. Accordingly, we tuned our automated synapse detection algorithm to exclude synapses that we were unable to accurately segment because they closely abut in z (see rule 8, above). 
Presumably, this trade off led to increased false negative rates, but reduced false positive rates that would have arisen from incorrectly merging two distinct synapses.

a

Slice SEP-GluA1, stain w/Homer $a b$ th of synapse identification

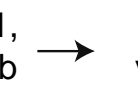

$$
2 p \text { in vitro, use in }
$$
vivo laser settings

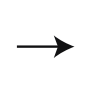

Mask, remove low intensity areas

Calculate \% SEP/Homer overlap (ground truth)
Auto. detect SEP and Homer puncta b Single, raw $2 p$ image plane

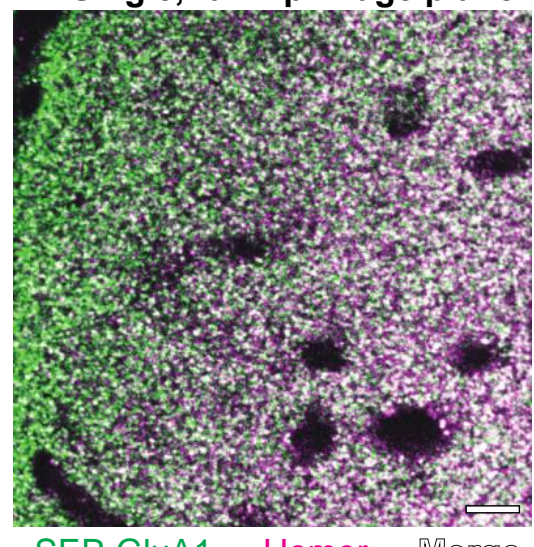

SEP-GluA1 Homer Merge

\section{d Auto. detect SEP/Homer}

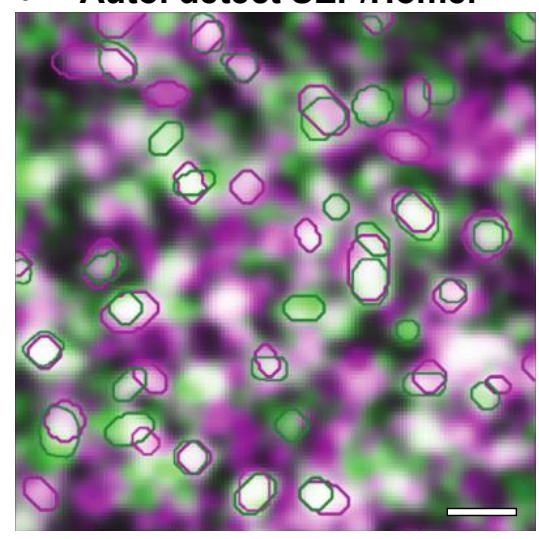

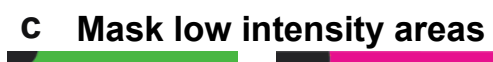

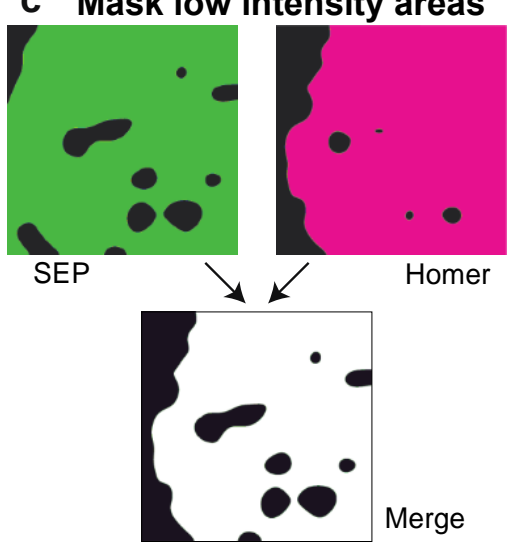

e Quantify SEP/Homer overlap

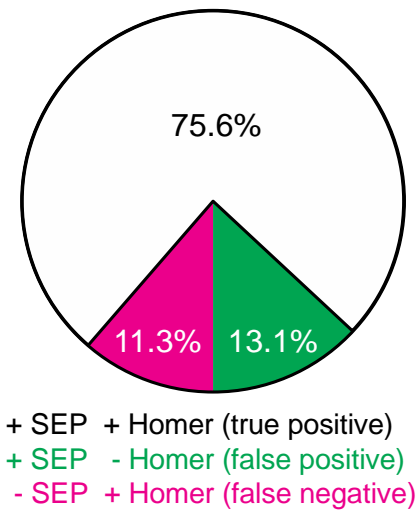

$\begin{array}{lll}\text { Figure } \quad 7 & \text { Supplement } 2 .\end{array}$ Schematic of in vitro automatic detection of Homer and SEPGluA1 puncta. a. Workflow to establish ground truth of synapse detection. b. Representative, raw image of in vitro $2 p$ signal of layer I SEP-GluA1 (green) barrel cortex stained with a Homer antibody (magenta); voxels displaying overlap of SEP-GluA1 and Homer are rendered in white. c. Schematic of masking to remove low-intensity regions. Regions lacking detectable SEP fluorescence were excluded by thresholding, removing areas with a $z$ score of less than -1 in a blurred image (see Methods). d. Zoomed-in image of raw in vitro $2 p$ signal, with overlaid automatic detections/segment-ations of SEP-GluA1 (green circles) and Homer (magenta circles). e. Quantification of ground truth for synapse detection. True positive rate reflects rate of overlap between SEP-GluA1 and Homer, false positive rate reflects rate of SEP-GluA1 detection without overlapping Homer, and false negative rate reflects rate of Homer detection without overlapping SEP-GluA1. A threshold of $>50 \%$ total voxel overlap defined overlap.

\section{SEP-GluA1 reports synaptic plasticity underlying sensory stimulation}

To demonstrate the utility of the SEP-GluA1 knockin line and our automated synapse detection algorithm, we investigated synaptic dynamics in barrel cortex during whisker stimulation. Mouse somatosensory cortex displays an exquisite somatotopic map, wherein each individual whisker is represented by a discrete cortical area. These so-called barrels provide an ideal tableau to investigate activity-dependent plasticity underlying encoding of sensory stimulation. Previous work has shown that whisker stimulation can induce NMDA receptor dependent LTP in layer 2/3 cells (Gambino et al., 2014; Holtmaat and Caroni, 2016; Zhang et al., 2015). To investigate how this sensory stimulation may be stored within vast synaptic networks, we surgically implanted cranial windows over barrel cortex in adult SEP-GluA1 mice 
(2-4 months old). Using optical intrinsic imaging during passive whisker stimulation, we identified barrels corresponding to the C2 and D3 whiskers (Fig. 8a). We imaged SEP-GluA1 fluorescence at high resolution within $100 \mu \mathrm{m}$ cubed volumes of layer I somatosensory cortex. By registering volumes to vasculature and other fiducial markers, we were able to longitudinally image the same cortical volumes in each barrel for 3-5 hours. To confine our analysis to precisely the same neural volumes throughout sensory stimulation, we further employed post-hoc rigid-body transformations to longitudinally align neural volumes.
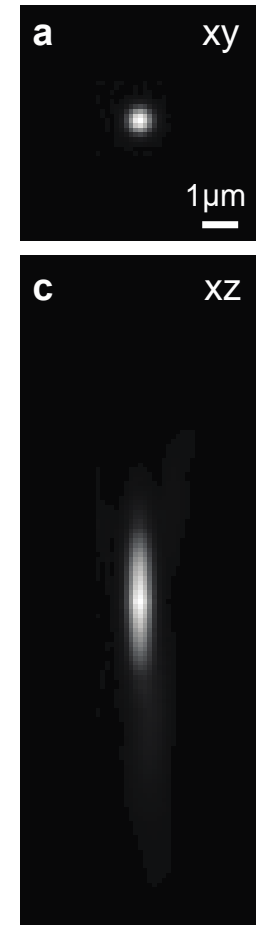

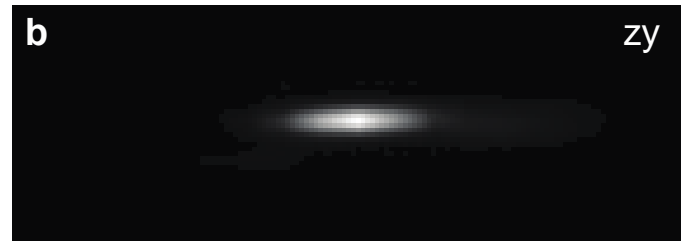

d

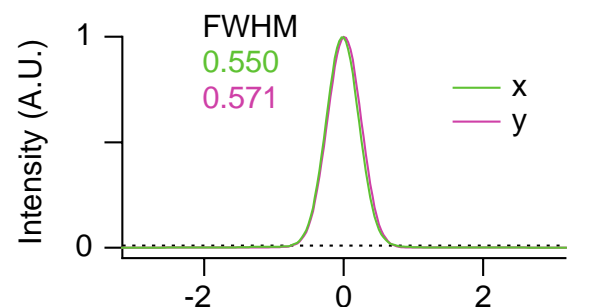

e

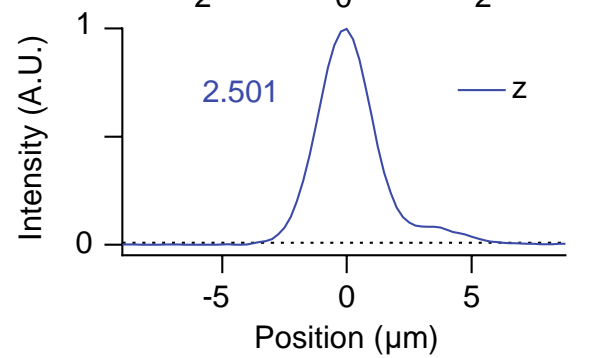

Figure 7 Supplement 3. Radial and axial PSF (point spread function) of $2 p$ microscope. a-c. Reconstructed PSF from averaged $2 p$ images of $1 \mu \mathrm{m}$ TetraSpeck microsphere $\mathrm{s}$ imaged at $910 \mathrm{~nm}$ in the GFP channel (scale bar: $1 \mu \mathrm{m})$. PSF fluorescence intensity profiles were visualized across the intensity center of the $z-(\mathbf{a}), y-$ (b), and x-axes (c). d-e. Linear profile of normalized intensity distribution shown in (a-c). The Full Width at Half Maximum (FWHM) of these profiles gives a direct estimate of the radial (d) and axial (e) PSF.

We were able to detect hundreds of thousands of synapses in each mouse and extracted their SEP-GluA1 fluorescence intensity at each time point. To investigate synaptic dynamics at baseline and during sensory stimulation, we delivered $10-\mathrm{Hz}$ mechanical stimulation exclusively to the $\mathrm{C} 2$ whisker in lightly anesthetized animals. Whereas the unstimulated barrel displayed a stable distribution of SEP-GluA1 intensity over time, the C2 barrel that received mechanical whisker stimulation displayed a significant rightward shift in the distribution of SEP-GluA1 intensity, consistent with increased synaptic SEP-GluA1 levels from induction of LTP (Fig. 8bd). This potentiation manifested as an increase in the mean SEP-GluA1 intensity (Fig. 8c) as well as a smaller but still significant increase in synapse size (Fig. 8d). The number of detected synapses in both the stimulated and unstimulated barrels was stable over time (Fig. 8e), suggesting that the observed plasticity was not expressed via net spinogenesis or pruning, but rather by a net potentiation of existing synapses. As this knockin line exclusively labels GluA1containing AMPARs, it is possible the changes we observed reflect subunit-specific dynamics rather than more general synaptic plasticity. 
a

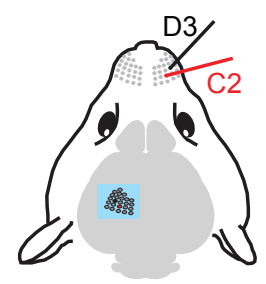

Image SEP-GluA1 expression during whisker stimulation

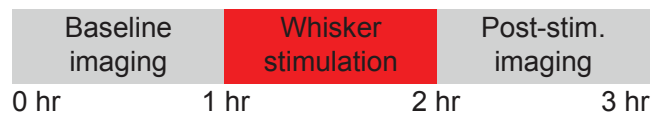

b

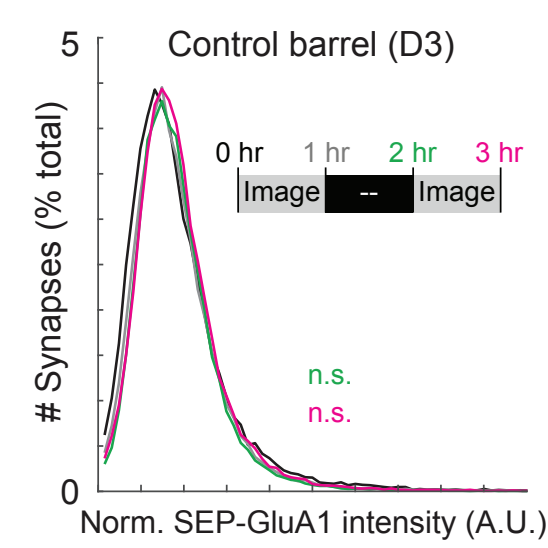

Distibution of SEP-GluA1 intensity

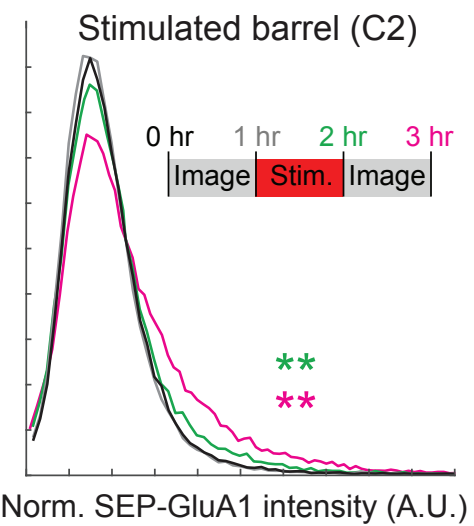

c

Stimulation potentiates SEP-GluA1 intensity

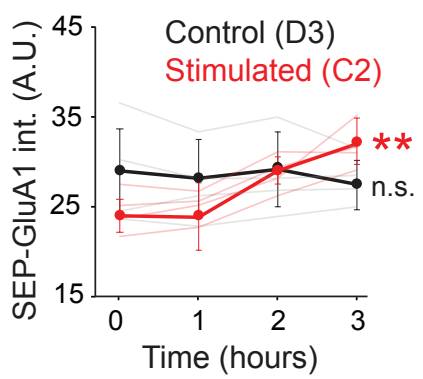

d

Stimulation increases synapse size

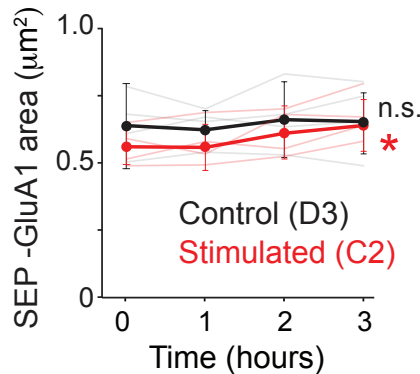

e Synapse number stable over time

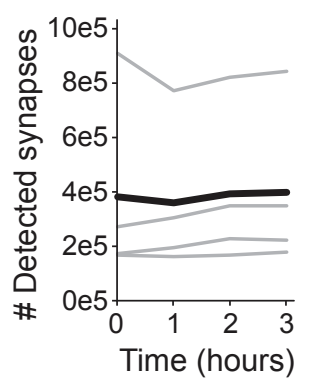

Figure 8. Tracking synaptic plasticity during sensory experience. a. Schematic of whisker stimulation and in vivo imaging of barrel cortex. The control unstimulated (D3) and stimulated barrel (C2) were imaged twice at baseline. The C2 whisker was mechanically stimulated with $10 \mathrm{~Hz}$ vibration for 1 hour, after which imaging of both barrels resumed. b. Distribution of normalized SEP-GluA1 intensity in barrel cortex over time in one representative homozygous SEP-GluA1 mouse. Left: distribution of SEP-GluA1 intensity was stable over time in the control, unstimulated D3 barrel. Right: significant rightward shift in SEP-GluA1 intensity in the C2 barrel following whisker stimulation (for $1 \mathrm{hr}$, between the 1-2 hour time points), indicating net synaptic potentiation. ${ }^{* *} p<0.01$, Mann-Whitney $U$ test relative to within-mouse baseline period (pooled 0 and 1 hour timepoints). c. Whisker-stimulation induces barrel-selective synaptic potentiation. Plot of mean SEP-GluA1 fluorescent intensity from all automatically detected SEP puncta over time in the control (black, D3) and stimulated (red, C2) barrel. $n=4$ mice, ${ }^{* *} p<0.01,2$-way ANOVA, comparing each imaging session to the two pooled baseline sessions ( 0 and $1 \mathrm{hr}$ ). Error bars represent standard deviation. d. Whisker-stimulation induces barrel-selective synapse enlargement. Plot of mean area of all automatically detected SEP puncta over time in the control (black, D3) and stimulated (red, C2) barrel. Synapse area was defined as the maximum area in a single 2D imaging plane for each automatically segmented SEP-GluA1 puncta. * $p<0.05$, 2-way ANOVA, comparing each imaging session to the two pooled baseline sessions ( 0 and $1 \mathrm{hr}$ ). e. Number of detected synapses was stable over time. Total synapse count from each individual mouse (thin grey) and mean ( $n=4$, thick black) are presented. 


\section{Discussion}

A central goal of neuroscience is to understand high-order cognitive functions in terms of their constituent components. Over the past 50 years, we have learned a great deal regarding the general role of synaptic plasticity in learning and memory. While seminal experiments have clearly implicated regulation of AMPARs as a central mechanism to modify the strength of synaptic communication between neurons (Andersen et al., 1977; Frey and Morris, 1997; Huganir and Nicoll, 2013; Malinow and Malenka, 2002; McNaughton et al., 1978), there is currently a dearth of methodologies to investigate how these molecular dynamics are distributed within vast networks of billions of synapses throughout the brain. For instance, patch-clamp recordings provide excellent spatial and temporal resolution, enabling investigation of how integration of specific synaptic inputs is dynamically tuned by plasticity (Spruston, 2008), but the scale of these recordings is limited to single neurons and performing them in behaving animals is challenging (Bittner et al., 2017; Epsztein et al., 2011). In vivo calcium imaging and highchannel-count electrophysiology offer superb spatial coverage, enabling investigation of neuronal activity within circuits of hundreds of neurons during behavior (Juavinett et al., 2019; Jun et al., 2017; Sofroniew et al., 2016), but these techniques lack the spatial resolution to study plasticity of individual synapses. In vivo structural imaging has provided valuable insights regarding how spine formation and elimination has been shown to contribute to neuronal development and synaptic plasticity (Bhatt et al., 2009; Holtmaat and Svoboda, 2009; Trachtenberg et al., 2002; Xu et al., 2009; Yang et al., 2009), though this method does not measure plasticity at existing spines. In addition, spine size has been reported to be proportional to synaptic strength (Matsuzaki et al., 2001), but this is at best an indirect readout of synaptic strength, and in certain conditions spine size and synapse strength are completely dissociated (Lee et al., 2012; Sdrulla and Linden, 2007; Tan et al., 2020; Zhang et al., 2015). Thus, our understanding of how the brain represents learning, memory, and behavior is constrained by currently available methodologies.

Here, we present a suite of novel tools and approaches that break through these constraints, enabling visualization of synaptic plasticity with molecular resolution at brain-wide scale in living animals. At the heart of this suite lies the newly generated SEP-GluA1 knockin mouse, a novel line that fluorescently labels all endogenous GluA1-containing AMPARs throughout the entire brain. This line enables direct investigation of the molecular dynamics underlying synaptic plasticity at any scale, from super-resolution synaptic imaging in primary cultures to circuit-level analyses of plasticity in acute slices to brain-region-wide imaging of synaptic strength in behaving animals. The sequence linking the SEP tag and AMPAR Nterminus is known to affect protein expression and proper postsynaptic targeting of the receptor, as previous attempts to fluorescently tag AMPARs at the N-terminus have been reported to result in impaired synaptic function (Diaz-Alonso and Nicoll, 2021; Diaz-Alonso et al., 2017). However, in our experiments, $\mathrm{N}$-terminal linkers were lengthened and optimized to increase the flexibility of the SEP tag, thereby limiting disruption of GluA1 function.

We conducted extensive validation of our novel knockin line, demonstrating that SEPGluA1 mice exhibit normal synaptic physiology, AMPA receptor trafficking, and general behavior that is indistinguishable from WT littermates, strongly supporting that our endogenous labeling strategy does not impair synaptic function in any detectable manner. Using both primary cultures and acute slices, we showed that the SEP-GluA1 line reports both increases and decreases in synaptic GluA1 content, and it is an effective tool to study several forms of AMPAR-mediated plasticity, including homeostatic scaling and long-term potentiation. Using $2 p$ glutamate uncaging, we clearly demonstrated that intensity of SEP fluorescence directly correlates with 
functional synaptic strength, indicating that this line can be used as an effective tool to study synaptic plasticity in vivo. Using FRAP, we demonstrated that SEP-GluA1-containing synapses display normal synaptic mobility and dynamics. Finally, we developed a computer-vision algorithm to automatically detect and segment extremely large numbers of endogenously labeled synapses across entire brain regions in living animals. Using these tools, we were able to longitudinally track synaptic plasticity encoding sensory stimulation with unprecedented spatial coverage and molecular resolution, producing the most detailed spatiotemporal map of behaviorally relevant synaptic plasticity to date. While the SEP-GluA1 line serves as an effective tool to investigate many forms of plasticity in vitro and in vivo, we are continuing to develop similar knockin and transgenic lines that similarly label GluA2-4, as well as other proteins of interest that may play key roles in synaptic transmission and plasticity. For example, it is important to consider that our approach to monitor SEP-GluA1 might favor the detection of changes in synapses preferentially undergoing plasticity rather than an absolute change in synaptic strength. Similar endogenous labeling strategies of other synaptic proteins would be useful to investigate the molecular mechanisms of nearly any behavior, including GluA1independent forms of plasticity (Frey et al., 2009) or disease models that display synaptic pathologies, such as SynGAP haploinsufficiency or Alzheimer's disease (Gamache et al., 2020; Sheng et al., 2012).

By fluorescently tagging endogenous GluA1-containing AMPARs and utilizing in vivo $2 p$ microscopy, we were able to directly visualize the functional strength of endogenous synaptic networks and track how they change during sensory stimulation. We demonstrated that mechanical stimulation of a single mouse whisker leads to increased synaptic GluA1 specifically in the cortical region corresponding to the stimulated whisker. This is consistent with previous studies showing NMDA-receptor-dependent LTP following whisker stimulation (Gambino et al., 2014; Holtmaat and Caroni, 2016; Zhang et al., 2015). While our proof-of-principle experiments clearly illustrate the power of this approach to image endogenous AMPARs and track widespread synaptic plasticity in vivo, these data represent only the tip of the iceberg. As our genetic labeling strategy illuminates all GluA1-containing synapses throughout the brain, this line enables investigation of synaptic plasticity from any brain region during any behavioral paradigm of interest. Further, this flexible tool is compatible with any electrophysiological method or other fluorescence-based imaging approaches, such as neuronal activity sensors (e.g. RCaMP) and genetic tagging of cell-types of interest (e.g. engram cells, Cre-lines, etc.). For example, by crossing the SEP-GluA1 knockin line with the Ai9 tdTomato reporter mouse and expressing Cre in a cell-type or neuronal circuit of interest, it is possible to specifically quantify changes in spine GluA1 expression of these neurons, as demonstrated in Fig. 6 and Fig 7c,d. Beyond visualizing fluorescently labeled cortical synapses through cranial windows, one could also use our tools to investigate synaptic dynamics in subcortical structures using cortical excavation, endoscopes, or fiber photometry.

More generally, this strategy to label endogenous synaptic receptors has several key advantages over previous approaches. Building upon insights gleaned by spine dynamics, in which the formation and elimination of dendritic spines has been shown to be involved in several forms of learning (Holtmaat and Svoboda, 2009; Xu et al., 2009; Yang et al., 2009), our approach additionally enables investigation of plasticity in existing synapses. Recently, similar genetic labeling strategies have been used to investigate other synaptic proteins, such as PSD-95 (Cane et al., 2014; Fortin et al., 2014; Gray et al., 2006; Zhu et al., 2018). These studies have revealed crucial details regarding how scaffolding and structural proteins contribute to dynamic synapse function. Here, we build upon these findings by directly imaging AMPARs, which are the principle functional unit of the synapse. While overexpression of SEP-labeled AMPARs has been 
previously used to investigate behaviorally relevant plasticity (Diering et al., 2017; El-Boustani et al., 2018; Miyamoto et al., 2021; Roth et al., 2019; Suresh and Dunaevsky, 2017; Tan et al., 2020; Zhang et al., 2015), the current study is the first to engage labeling of endogenous receptors, which much more faithfully reports the direct physiological mechanisms of plasticity.

Recently, deep-learning-based systems have achieved state-of-the-art performance in analyzing microscopy images (Moen et al., 2019). Many different architectures have been used, including our work detecting tau tangles with sliding windows to annotate single pixels (Tward et al., 2020), UNET (Ronneberger et al., 2015) for annotating larger blocks which has been implemented in FIJI (Falk et al., 2019), and other elaborations such as VNET (Milletari et al., 2016). Typically, trained networks assign class probabilities to each pixel, which are collected into larger objects based on connected components or watershed approaches available in standard packages, such as FIJI (Schindelin et al., 2012). A caveat of the deep learning approach is that these methods require a large quantity of high-quality training data, which is not available for new image types such as those used in our work. Another approach to annotating microscopy images has been to employ interactive methods. For example, the framework developed by ilastic allows users to annotate small regions, and constructs a random forest model based on simple features (brightness, edges, texture) to extend the per pixel annotations to large images (Berg et al., 2019). The annotated regions can be modified or extended until optimal performance is achieved. Importantly, this approach works well in situations where annotation is easy "by eye", but is challenging at scale. We found that none of these approaches performed as well as our rules-based, template-matching approach to automatically detect and segment SEP-GluA1-containing synapses.

To achieve the goal of understanding plasticity at individual synapses during complex behaviors and learning, it is crucial to track individual synapses over time. Here, we have tracked the same population of spines during whisker stimulation (Fig. 8) and have also shown that by adding a cytosolic fluorescent protein, it is possible to achieve longitudinal imaging with the ability to track individual synapses across days (Fig. 6a and C). In future studies, we will expand on our computational approach to enable alignment, registration, and tracking of millions of individual synapses throughout the entire process of learning. While our current imaging experiments were performed in lightly anesthetized mice, our previous work has demonstrated the feasibility to visualize and track SEP-GluA1 in individual spines in head-fixed awake behaving mice (Tan et al., 2020) with the same reliability and resolution as in anesthetized mice, suggesting that imaging awake SEP-GluA1 knockin mice will be feasible.

In conclusion, we aim to fundamentally advance our understanding of the synaptic basis of behavior, moving beyond merely studying synaptic plasticity in single neurons, seeking instead to explore dynamic modulation of the complete synaptome during learning and memory. The tools presented here make this goal achievable. 


\section{Supplemental Videos}

Movie 1. Representative in vivo $2 p$ imaging volume of SEP-GluA1 knockin. The imaging volume displayed is $98 \times 98 \times 130 \mu \mathrm{m}$ section of layer I barrel cortex, with SEP-GluA1 synapses in green and a sparse cell fill in magenta. Overlap between green/magenta voxels is rendered in white. The movie begins at the pial surface, moving ventrally towards layer II/III, with depth indicated in the lower right. Note the high density of green puncta, each corresponding to a single GluA1-containing synapse. Punctate synaptic labels are observed uniformly up to a depth of $\sim 100 \mu \mathrm{m}$, below which the signal begins to degrade, likely due to light scattering. In this SEP-GluA1 x Ai9 mouse, a random subset of layer II/III pyramidal cells was filled using a dilute (1:20k) AAV-CaMK2-Cre virus. Voxel size is $0.096 \times 0.096 \times 1 \mu \mathrm{m}$. Resolution is $1024 \times 1024$ pixels in $X Y$ and a $1 \mu \mathrm{m}$ step size. Images were median filtered with a radius of 1 and contrast enhanced. Scale bar is $10 \mu \mathrm{m}$.

Movie 2. 3D rendering of filled dendrite with SEP-GluA1 puncta in spines. The imaging volume displayed is a $30 \times 30 \times 15 \mu \mathrm{m}$ section of layer I barrel cortex. Note the enrichment of SEPGluA1 puncta (green) in dendritic spines (magenta) and the paucity of SEP signal in the dendritic shaft. There are many green puncta outside of the filled cell, likely corresponding to GluA1-containing synapses in spines of unlabeled cells. 3D volume was rendered using Imaris. Dynamic scale bar in lower left.

Movie 3. 3D rendering of SEP-GluA1 puncta in layer I barrel cortex. $35 \times 50 \times 15 \mu \mathrm{m}$ imaging volume. Note the extreme density of green puncta, each corresponding to a single GluA1containing synapse. Rendered using Imaris. Dynamic scale bar in lower left. 


\section{Methods and Materials}

\section{Neuronal culture}

Mouse embryonic (E18) cortical/hippocampal neurons were plated on poly-L-lysine coated tissue culture dishes/glass coverslips at a density of $65,000 \mathrm{cells} / \mathrm{cm}^{2} / 37,500 \mathrm{cells} / \mathrm{cm}^{2}$ in NM5 medium (neurobasal media (Invitrogen) supplemented with 2\% B-27, 2 mM Glutamax, $50 \mathrm{U} / \mathrm{mL}$ PenStrep, and $5 \%$ horse serum (Invitrogen)) and grown in NM0 medium (neurobasal media (Invitrogen) supplemented with 2\% B-27, 2 mM GlutaMAX (50 U/mL, PenStrep)). Cultured cortical neurons/hippocampal neurons were fed twice/once per week. To induce synaptic scaling, cortical neurons were treated with bicuculline $(20 \mu \mathrm{M})$ or tetrodotoxin (TTX, $1 \mu \mathrm{M})$ at DIV 11-13 for 48 hours. Hippocampal neurons were used at DIV 19-22 for glutamate uncaging.

\section{Surface biotinylation}

Neurons were rinsed with ice-cold PBSCM $\left(1 \times \mathrm{PBS}, 1 \mathrm{mM} \mathrm{MgCl}_{2}, 0.1 \mathrm{mM} \mathrm{CaCl}_{2}, \mathrm{pH}\right.$ 8.0) once and then incubated with Sulfo-NHS-SS-biotin $(0.5 \mathrm{mg} / \mathrm{ml}$, Thermo Scientific) for 30 minutes at $4^{\circ} \mathrm{C}$. Residual unreacted biotinylation reagent was washed out with PBSCM and quenched by $20 \mathrm{mM}$ glycine twice for 5 minutes. Neurons were lysed in lysis buffer [PBS containing $50 \mathrm{mM}$ $\mathrm{NaF}, 5 \mathrm{mM}$ sodium pyrophosphate, $1 \%$ NP-40, $1 \%$ sodium deoxycholate, $0.02 \%$ SDS, and protease inhibitor cocktail (Roche)]. $20 \mu \mathrm{g}$ lysates were incubated overnight with NeutraAvidin agarose beads (Thermo Scientific) and then were washed with lysis buffer four times. Biotinylated proteins were eluted using $2 \times$ SDS loading buffer. Surface proteins were then subjected to SDS-PAGE and analyzed by Western blot.

\section{PSD fractionation}

Mouse hippocampus tissues were homogenized in buffer [320mM sucrose, $5 \mathrm{mM}$ sodium pyrophosphate, $1 \mathrm{mM}$ EDTA, 10mM HEPES pH 7.4, 200nM okadaic acid, protease inhibitor cocktail (Roche)] using a 26-gauge needle. Homogenate was centrifuged at $800 \times \mathrm{g}$ for 10 minutes at $4^{\circ} \mathrm{C}$ to yield $\mathrm{P} 1$ (nuclear) and S1 (post-nuclear). S1 was centrifuged at 20,000 $\times \mathrm{g}$ for 20 minutes to yield P2 (membrane) and S2 (cytosol). P2 was then resuspended in water adjusted to $4 \mathrm{mM}$ HEPES $\mathrm{pH} 7.4$ followed by 30 minutes' agitation at $4^{\circ} \mathrm{C}$. Suspended $\mathrm{P} 2$ was centrifuged at $25,000 \times \mathrm{g}$ for 20 minutes at $4^{\circ} \mathrm{C}$. The resulted pellet was resuspended in $50 \mathrm{mM} \mathrm{HEPES} \mathrm{pH}$ 7.4 , mixed with an equal volume of $1 \%$ triton $\mathrm{X}-100$, and agitated at $4^{\circ} \mathrm{C}$ for 10 minutes. The PSD fraction was generated by centrifugation at $32,000 \times \mathrm{g}$ for 20 minutes at $4^{\circ} \mathrm{C}$.

\section{Cell-culture immunohistochemistry and confocal imaging}

Cultured hippocampal neurons were fixed for $20 \mathrm{~min}$ in PBS containing $4 \%$ paraformaldehyde (PFA)/4\% sucrose and rinsed with PBS. Neurons were blocked, permeabilized and incubated with primary antibodies in GDB buffer (15 mM phosphate buffer $(\mathrm{pH} 7.4)$ containing $0.1 \%$ gelatin, $0.3 \%$ Triton $\mathrm{X}-100$, and $0.25 \mathrm{M} \mathrm{NaCl}$ ) at $4^{\circ} \mathrm{C}$ overnight. Coverslips were washed with PBS before the neurons were incubated with secondary antibodies in GDB buffer for $1 \mathrm{~h}$ at room temperature. After washing with PBS and water, coverslips were mounted onto glass slides using Permafluor (Fischer Scientific). Images were obtained using an LSM880 laser scanning confocal microscope (Zeiss). The following antibodies were used: antiGluA1 C-terminal pAb (JH4294, made in house), anti-PSD95 mAb (NeuroMab), anti-GFP pAb (ab13970 , Abcam), Alexa Fluor 488 goat anti-chicken (Thermo Fisher Scientific), Alexa Fluor 405 goat anti-mouse (Thermo Fisher Scientific), and Alexa Fluor 647 goat anti-rabbit (Thermo Fisher). 


\section{Electrophysiological recordings}

Whole-cell voltage-clamp recordings were performed in CA1 pyramidal neurons of acute hippocampal slices from 3-4-weeks-old paired littermates of mice by an experimenter blind to genotype. Slices were prepared in ice-cold oxygenated dissection buffer containing the following (in $\mathrm{mM}$ ):210 Sucrose, 7 Glucose, 26.2 NaHCO3, $2.5 \mathrm{KCl}, 1 \mathrm{NaH} 2 \mathrm{PO} 4,7 \mathrm{MgSO}$. For all recordings, slices were perfused in ACSF $(119 \mathrm{mM} \mathrm{NaCl}, 26.2 \mathrm{mM} \mathrm{NaHCO} 3$ and $11 \mathrm{mM}$ glucose,

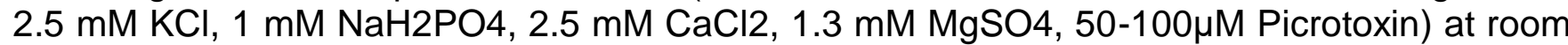
temperature. Neurons were patched by glass pipettes (3-5 $M \Omega$ ) which were filled with internal solution (115 mM Cs-MeSO3, $0.4 \mathrm{mM}$ EGTA, $5 \mathrm{mM}$ TEA-Cl, $2.8 \mathrm{mM} \mathrm{NaCl}, 20 \mathrm{mM}$ HEPES, 3 $\mathrm{mM}$ Mg-ATP, $0.5 \mathrm{mM} \mathrm{Na2-GTP,} 10 \mathrm{mM}$ Na Phosphocreatine, $5 \mathrm{mM}$ QX-314). mEPSC recordings were performed in the ACSF in presence of $1 \mu \mathrm{M} \mathrm{TTX}$, and cells were hold at $-70 \mathrm{mV}$. Data from 5-10 min after break-in were used for mEPSC analysis. For rectification and LTP experiments, EPSCs were elicited at $0.1 \mathrm{~Hz}$ by electrical stimulation $(0.1 \mathrm{~ms}, 8-20 \mu \mathrm{A})$ via a stimulating electrode positioned in stratum radiatum. During rectification measurements, $100 \mathrm{nM}$ Spermine was added into the internal solution and cells were held at -60 to $60 \mathrm{mV}$ before liquid junction modification. Each data point at each potential was averaged by 5-10 EPSCs. LTP was induced by a train of 200 pulses at $2 \mathrm{~Hz}$ paired with $0 \mathrm{mV}$ depolarization. Data are presented as EPSC amplitude averaged at 1-min intervals and normalized to baseline. Signals were measured with MultiClamp 700B amplifier and digitized at $10 \mathrm{kHz}$ by using a Digidata $1440 \mathrm{~A}$. Data acquisition were performed with pClamp 10.5 software. Access resistance (Ra) was monitored throughout the recording. Cells in which the $\mathrm{Ra}>20 \mathrm{M} \Omega$ or Ra varied by more than $20 \%$ were discarded.

\section{Mouse behavior}

Behavioral testing was performed in homozygous SEP-GluA1 mice ( 9 females and 7 males) and WT (9 females and 9 males) littermate controls, aged 6-10 weeks. Animals were housed in a holding room on a reverse light cycle, and testing was conducted during the dark (i.e., active) phase. All behavioral experiments were approved by the Johns Hopkins Johns Hopkins Animal Care and Use Committee.

Locomotor activity was assessed by placing animals in an illuminated open arena $(40 \times 40 \mathrm{~cm})$ and measuring the number of infrared beam breaks during a 30-minute session (San Diego Instruments Inc.). Anxiety was assessed using an elevated plus maze $(66 \mathrm{~cm}$ long and $5 \mathrm{~cm}$ wide; San Diego Instruments Inc.), consisting of two closed arms and two open arms suspended $54 \mathrm{~cm}$ above the ground. Immediately before testing, animals were placed, individually, into a clean cage for 5 minutes. Animals were placed onto the center of the elevated plus maze facing an open arm and allowed to explore for 5 minutes. Animal position was tracked using ANYmaze software (Stoelting, IL).

Spatial short-term memory was assessed by testing spatial novelty preference using a $Y$ maze. The Y-maze was made of clear plexiglass (each arm $38 \mathrm{~cm}$ long; San Diego Instruments Inc.) and surrounded by distal spatial cues. A mixture of clean and dirty sawdust (ratio 2:1) was added to the bottom of the maze to promote exploration of the maze. The dirty sawdust was collected from other cages mice, of the same sex as the animals being tested. Immediately before testing, animals were placed, individually, into a clean cage for 5 minutes. The test was split into exposure and test phases. During the exposure phase, one of the Y-maze arms was blocked (counterbalanced for genotype) and animals were allowed to explore two arms of the maze for 5 minutes. After this exposure phase, animals were gently removed from the maze and returned to the temporary holding cage for 1 minute. During which the sawdust was redistributed, and all arms of the maze were made available. For the test phase, mice were re- 
exposed to the maze and allowed to explore all arms for 2 minutes. Testing was conducted by an experimenter blind to genotype of the mice being tested. Statistical comparisons were made using SPSS(IBM). Sex and genotype were used as between subject variables.

\section{$2 p$ glutamate-uncaging}

Cultured mouse cortical neurons (10:1 mixture of WT and homozygous SEP-GluA1) were plated at E18 and imaged on DIV 16-18. Neurons were perfused in a modified HEPES-based ACSF solution, consisting of (in mM): $140 \mathrm{NaCl}, 5 \mathrm{KCl}, 10$ glucose, $10 \mathrm{HEPES}, 2 \mathrm{CaCl}_{2}, 1 \mathrm{MgCl}_{2}$, $1 \mathrm{TTX}$, and $2.5 \mathrm{mM}$ MNI-caged-L-glutamate (Tocris), $\mathrm{pH}=7.30$ and 310-316 mOsm. Recordings were made at room temperature in recirculated ACSF (3 mL/min). Recording pipettes were fabricated (Flaming/Brown Micropipette Puller, Sutter Instruments) from borosilicate capillary glass (Sutter, 4-6 M $\mathrm{M}$ open-tip resistance) and filled with (in mM): $115 \mathrm{CsMeSO} 4,2.8 \mathrm{NaCl}, 5$ TEACI, 0.4 EGTA, 20 HEPES, 3 MgATP, 0.5 NaGTP, 10 NaPhosphocreatine, and 2.5 QX-314, $\mathrm{pH}=7.32$ and $306 \mathrm{mOsm}$, and containing a 1\% Alexa-594 dye (Tocris). Whole-cell voltageclamp recordings were made using a MultiClamp 700B amplifier and Digidata 1440A digitizer (Axon Instruments).

Neurons were imaged with a 20X/1.0 NA water-immersion objective (Zeiss) and a custom-built $2 \mathrm{p}$ microscope (MOM system, Sutter Instruments) controlled by Scanlmage (Vidrio Technologies, Ashburn, VA). Dendritic morphology was visualized using an Alexa dye, delivered by the patch pipette). SEP-GluA1 and red cell fill were excited at $910 \mathrm{~nm}$ using a tunable Ti:sapphire laser (Coherent, Santa Clara, CA). Images were acquired at 1024x1024 resolution and slices within z-stacks spaced every $0.5 \mu \mathrm{m}$. A second $2 p$ laser (Spectra Physics, Santa Clara, CA) was used to uncage glutamate (1 ms pulse) onto visually identified spines at a wavelength of $730 \mathrm{~nm}$ and a power of $20 \mathrm{~mW}$ at the objective back aperture. Uncaging position was controlled using custom software developed in our lab (ScanStim), which provided means to correct for chromatic aberration between the imaging and uncaging beam. The offset between the imaging and uncaging $2 p$ lasers was directly measured and corrected on a monthly basis. To measure the glutamate-uncaging-evoked excitatory postsynaptic current (UEPSC), we used pClamp (Axon Instruments) to synchronize triggering of the uncaging laser with voltage-clamp recordings. To minimize the effect of electrotonic filtering caused by variable numbers of branch points between the site of dendritic uncaging and the somatic recording pipette, we uncaged exclusively onto spines of secondary dendrites, located 95-160 $\mu \mathrm{m}$ from the cell body. We uncaged on 4-8 spines/dendritic segment and 1-3 dendritic segments/neuron. To quantify the SEP-GluA1 and cell-fill signals, we manually drew ROls around visually identified spines, summed the fluorescent intensity of five adjacent Z-sections (each separated by $0.5 \mu \mathrm{m}$ ), and subtracted size-matched neighboring background ROls. Representative images shown in figures were median filtered and contrast enhanced. The uncaging-LTP-induction stimulus consisted of 30 pairings of glutamate uncaging (1ms pulse of $730 \mathrm{~nm}$ laser at $0.5 \mathrm{~Hz}$ ) and postsynaptic depolarization ( $0 \mathrm{mV}$ for $0.5 \mathrm{sec}$, beginning concurrently with uncaging pulse). Spines were imaged every 5 minutes and synaptic strength was probed by measuring the uEPSC amplitude of each identified spine every 1 minute.

\section{Cranial window surgery and viral injection}

Mice were anesthetized (2\% isoflurane) and implanted with a $3 \times 3 \mathrm{~mm}$ cranial window (Potomac Photonics) over the barrel cortex region of somatosensory cortex at 2-3 months of age. Windows were sealed and custom-made metal head bars attached using dental cement (Metabond; Edgewood, NY). In a subset of experiments, an AAV-CaMKII-cre virus (Addgene/Penn Vector) was injected into barrel cortex (1:10k-1:50k dilution, 100-150 nL, 0.25- 
$0.3 \mathrm{~mm}$ deep) of double homozygous SEP-GluA1 x Ai9 reporter mice to sparsely label L2/3 pyramidal neurons with a tdTomato cell fill. $10 \mathrm{mg} / \mathrm{kg}$ of extended-release Buprenorphine (ZooPharm) was administered before surgery and mice were observed for 3 days following surgery. Mice were allowed to recover from surgery for at least 2 weeks before commencing in vivo imaging. All surgical procedures were approved by the Johns Hopkins Johns Hopkins Animal Care and Use Committee.

\section{Optical-intrinsic and in vivo $2 p$ imaging}

Optical intrinsic imaging was used to map select barrels within somatosensory cortex as previously described (Zhang et al., 2015). Briefly, we mechanically stimulated the individual C2 and D3 whiskers using a custom-built piezo driver at $10 \mathrm{~Hz}$ and used optical intrinsic imaging to identify the corresponding barrel fields. Mice were anesthetized and maintained on $0.5 \%$ isoflurane supplemented by xylazine $(13 \mathrm{mg} / \mathrm{kg})$. Optical images of barrel cortex were acquired at $30 \mathrm{~Hz}$ using a CCD camera (Grasshopper GS3-U3-23S6M-C under red LED light $(630 \mathrm{~nm})$ with a $2.5 \times / 0.075$ numerical aperture (NA) objective (Zeiss). Images were collected, averaged (across 30 trials), Gaussian filtered $(\sigma=10 \mu \mathrm{m})$, and baseline subtracted. Widefield images of both barrels were acquired and vasculature was used to align subsequent $2 p$ imaging of the same regions.

In vivo $2 p$ images were acquired from lightly anesthetized mice $(13 \mathrm{mg} / \mathrm{kg}$ xylazine and $0.5 \%$ isoflurane) using a custom-built, $2 p$ laser-scanning microscope controlled by Scanlmage (Vidrio, Ashburn, VA) and a 20×/1.0 NA water-immersion objective lens (Zeiss). SEP-GluA1 (green) and tdTomato cell fill (red) were both excited at $910 \mathrm{~nm}$ with a Ti:sapphire laser (SpectraPhysics, $20 \mathrm{~mW}$ power at objective back-aperture). Green and red fluorescence signals were acquired simultaneously and separated by a set of dichroic mirrors and filters (ET525/50m for green channel, ET605/70m for red channel, Chroma). Image stacks were acquired at 1,024 × 1,024 pixels with a pixel size of $0.096 \mu \mathrm{m}$ in $\mathrm{XY}$, with a z-step of $1 \mu \mathrm{m}$. Representative images shown in figures were median filtered (1-pixel radius) and contrast enhanced.

\section{Fluorescence recovery after photobleaching in vivo}

To longitudinally image the same populations of SEP-labeled synapses throughout FRAP, imaging volumes ( $1024 \times 1024$ pixels in XY, $15 \mu \mathrm{m}$ in z with $1 \mu \mathrm{m}$ steps) were manually aligned before each time point using the sparse tdTomato cell fill as a guide. Photobleaching of spines was achieved with repetitive xy scanning of specific regions of interest ( 22 $\times 22$ pixels) defined at the center plane of the image stack using ROI Group Editor in Scanlmage. Bleached subregions were excited at $910 \mathrm{~nm}$ with high-intensity illumination $(20-30 \%)$ with a Ti:sapphire laser (Coherent, $15-100 \mathrm{~mW}$ of power delivered to the objective back-aperture) at a dwell time of $\sim 3 \mu \mathrm{sec} / \mathrm{pixel}$ and 7 iterations. For each experiment $\sim 5-10$ spines were bleached at a time.

Frame alignment and averaging within each plane in the Z-stacks were performed using a rigid registration custom script, and images at different time points were aligned using StackGPS (https://github.com/ingiehong/StackGPS) in MATLAB. Fluorescence intensity values were measured in ImageJ. Circular regions of interest were defined around bleached and unbleached control spines and signal intensity was measured as the average of 3 planes centered on each spine. Values were background subtracted. Baseline fluorescence was normalized to 1 and the signal intensity of the bleached spines was normalized to the averaged signal intensity of the unbleached spines on the same image. FRAP was calculated as the fluorescence increase between time 0 , immediately after photobleaching, and the indicated time points. Only spines that maintained stable levels of tdTomato signal in all imaging sessions after recovering from photobleaching (intensity signal above maximum photobleaching induced in that 
spine at time 0) were included in the analysis. Graphing and curve fitting were performed in Prism 6 (GraphPad software). Symbols represent mean and error bars represent standard error of the mean (SEM). Curve fitting of fluorescence recovery from 0 to 30min was performed using nonlinear regression to fit an exponential one phase decay curve defined by $Y=(Y 0$ Plateau $)^{\star} \exp \left(-k^{*} x\right)+$ Plateau, where Plateau is the maximum fluorescence, $Y 0$ is starting fluorescence, $k$ is the rate constant of recovery (minutes-1), and $x$ is time (min). Outlier removal was performed using the ROUT method with false detection rate $Q=1 \%$. Solid line in curve fitting represents best fit curve and shaded area represents the 95\% confidence interval of the best fit.

\section{Automatic synapse detection and segmentation}

We defined synapses as regions with bright centers and dark surrounds, based on the following method. Images were blurred with a Gaussian kernel of standard deviation $5 \times 5 \times 1$ pixels, and all local maxima were considered to be candidates for synapses. Candidates less than 3 pixels from each other were removed using a farthest first traversal (Hochbaum, 1985; Rosenkrantz, 1977). A family of templates (Brunelli, 2009) were defined using square regions with a radius of 32 pixels. The foreground was described by ellipses containing between 20-150 pixels, 4 roundnesses (ratio of larger to smaller semimajor axes lengths) from 1 to 2.5 , and 12 angles from 0 to 2 pi. A background region of width 3 pixels was identified surrounding the ellipse. For each candidate synapse, a signal to noise ratio (SNR) was calculated to determine the most likely template: mean pixel intensity of foreground, minus mean of background, divided by the standard deviation of the region. The template which maximized SNR was associated to this candidate. Regions lacking detectable SEP fluorescence, such as blood vessels and cell bodies, were excluded by thresholding, removing areas with a z score of less than - 1 in a blurred image. Based on visual examination, these regions were most likely interneuron cell bodies or blood vessels.

Candidates were accepted as synapses only if all five of the following conditions were met: (1) their associated template had a size between 20 and 150 pixels (in a single z-plane; (2) ovacity between 1 and 2.5; (3) SNR greater than the 90 th percentile of 300 randomly selected locations; (4) SNR was not reduced by more than 33\% when averaging 2 adjacent slices; (5) SNR was not increased by averaging 7 adjacent slices. The fourth criterion was chosen because synapses span more than one slice, whereas noise does not, and the fifth was chosen because artifacts (e.g., autofluorescence) tend to span many more slices than synapses do. Candidate synapses in adjacent Z-planes that overlapped in XY were merged into a single, 3D synapse volume. To be finally considered a valid synapse, two further criteria were required: (1) 3D volumes must contain XY-overlapping putative synapses on 2-6 adjacent planes in $\mathrm{z}$.

Detection code was written in python using numpy and is made available in the form of a Jupyter notebook at https://github.com/twardlab/synapse labeling.

We defined agreement between two annotated sets of synapses, A and B, as follows. For each synapse in set $A$, we identified any overlapping synapses in B. If more than one overlapped, we chose one which overlapped by the largest amount. If there were no overlapping synapse, or the overlapping synapse in B overlapped by less than $50 \%$ the size of the synapse in $A$, this was considered a disagreement. Otherwise, voxel overlap of more than $50 \%$ was considered agreement. The accuracy between $A$ and $B$ was defined as the fraction of synapses that agreed. The accuracy between $B$ and $A$ was defined by reversing the roles of $A$ and $B$ above. This definition is not symmetric, and the two agreements are generally close but not equal. In each relevant figure, we report the average $A-B$ and $B-A$ agreement. 


\section{Ground-truth synapse detection using immunohistochemistry}

To label, visualize, and detect an independent synapse channel to which to compare SEP-GluA1, we perfused 10-week-old homozygous SEP-GluA1 mice with $4 \%$ paraformaldehyde, made 100- $\mu \mathrm{m}$-thick slices of barrel cortex, and stained for the postsynaptic density protein Homer using a polyclonal antibody (Synaptic Systems) and an Alexa-555 secondary antibody. Stained tissue was imaged with the same $2 p$ microscope and light path as used for in vivo imaging, using identical settings for excitation wavelength $(910 \mathrm{~nm})$, laser power, PMT gain, scan speed, and all other acquisition settings. Green (SEP-GluA1) and red (Homer) channels were separately analyzed using our in vivo automatic synapse detection algorithm. Ground truth for synapse detection was defined as the rate of overlap between SEP-GluA1 and Homer puncta, false positive was defined as the rate of SEP-GluA1 detection without overlapping Homer, and false negative was defined as the rate of Homer detection without overlapping SEP-GluA1. A threshold of $>50 \%$ shared voxels was used to assess overlap for all comparisons. 


\section{Acknowledgements}

ARG is supported by R21 AG063193. ARG and DJT are supported by Kavli Distinguished Postdoctoral Fellowships. ARG, AMB, and RLH are supported by R01 MH123212. AMB is supported by K99MH124920. ARG, JTV, and RLH are supported by a Schmidt Science Nascent Innovation Grant. ACS is supported by a Kavli Distinguished Graduate Fellowship. We thank members of the Huganir Lab for helpful comments and discussion.

\section{Contributions}

ARG designed and performed the immunohistochemistry, live confocal imaging, $2 p$ glutamate uncaging, whole-cell patch-clamp recordings, in vivo $2 p$ imaging and whisker stimulation; analyzed data; prepared figures and wrote the manuscript in collaboration with all authors. RHR designed and performed the immunohistochemistry, in vivo $2 p$ imaging, and whisker stimulation. HLT designed and performed Western blots, surface staining, and homeostatic scaling. QZ designed and performed mini recordings and slice-physiology LTP experiments. AMB designed and performed immunohistochemistry and behavior experiments. ELO designed and performed in vivo $2 p$ imaging and FRAP experiments. $\mathrm{IH}$ wrote code for rigid-body transformation of in vivo imaging datasets. ACS designed, performed, and analyzed data for localizing SEP puncta to dendritic spines, in collaboration with ARG. RCJ made the knockin mouse line and performed Northern blots. DJT designed and wrote code for automatic synapse analysis, in collaboration with ARG. JTV, MIM, and RLH contributed to study design and provided funding for the work. All authors edited and approved the manuscript.

\section{Ethics declarations}

Dr. Miller is a joint owner of AnatomyWorks. Dr. Miller's relationship with Anatomy-Works is being handled under full disclosure by the Johns Hopkins University. The other authors declare no competing interests. 


\section{References}

Andersen, P., Sundberg, S.H., Sveen, O., and Wigstrom, H. (1977). Specific long-lasting potentiation of synaptic transmission in hippocampal slices. Nature 266, 736-737.

Anggono, V., and Huganir, R.L. (2012). Regulation of AMPA receptor trafficking and synaptic plasticity. Curr Opin Neurobiol 22, 461-469.

Araki, Y., Zeng, M., Zhang, M., and Huganir, R.L. (2015). Rapid dispersion of SynGAP from synaptic spines triggers AMPA receptor insertion and spine enlargement during LTP. Neuron 85, 173-189.

Ashby, M.C., De La Rue, S.A., Ralph, G.S., Uney, J., Collingridge, G.L., and Henley, J.M. (2004). Removal of AMPA receptors (AMPARs) from synapses is preceded by transient endocytosis of extrasynaptic AMPARs. J Neurosci 24, 5172-5176.

Ashby, M.C., Maier, S.R., Nishimune, A., and Henley, J.M. (2006). Lateral diffusion drives constitutive exchange of AMPA receptors at dendritic spines and is regulated by spine morphology. $J$ Neurosci 26 , 7046-7055.

Bannerman, D.M., Deacon, R.M., Brady, S., Bruce, A., Sprengel, R., Seeburg, P.H., and Rawlins, J.N. (2004). A comparison of GluR-A-deficient and wild-type mice on a test battery assessing sensorimotor, affective, and cognitive behaviors. Behav Neurosci 118, 643-647.

Bats, C., Groc, L., and Choquet, D. (2007). The interaction between Stargazin and PSD-95 regulates AMPA receptor surface trafficking. Neuron 53, 719-734.

Berg, S., Kutra, D., Kroeger, T., Straehle, C.N., Kausler, B.X., Haubold, C., Schiegg, M., Ales, J., Beier, T., Rudy, M., et al. (2019). ilastik: interactive machine learning for (bio)image analysis. Nat Methods 16, 1226-1232.

Berryer, M.H., Hamdan, F.F., Klitten, L.L., Moller, R.S., Carmant, L., Schwartzentruber, J., Patry, L., Dobrzeniecka, S., Rochefort, D., Neugnot-Cerioli, M., et al. (2013). Mutations in SYNGAP1 cause intellectual disability, autism, and a specific form of epilepsy by inducing haploinsufficiency. Hum Mutat 34, 385-394.

Bhatt, D.H., Zhang, S., and Gan, W.B. (2009). Dendritic spine dynamics. Annu Rev Physiol 71, 261-282.

Bittner, K.C., Milstein, A.D., Grienberger, C., Romani, S., and Magee, J.C. (2017). Behavioral time scale synaptic plasticity underlies CA1 place fields. Science 357, 1033-1036.

Bliss, T.V., and Lomo, T. (1973). Long-lasting potentiation of synaptic transmission in the dentate area of the anaesthetized rabbit following stimulation of the perforant path. J Physiol 232, 331-356.

Boerner, T., Bygrave, A.M., Chen, J., Fernando, A., Jackson, S., Barkus, C., Sprengel, R., Seeburg, P.H., Harrison, P.J., Gilmour, G., et al. (2017). The group II metabotropic glutamate receptor agonist LY354740 and the D2 receptor antagonist haloperidol reduce locomotor hyperactivity but fail to rescue spatial working memory in GluA1 knockout mice. Eur J Neurosci 45, 912-921.

Brunelli, R. (2009). Template Matching Techniques in Computer Vision: Theory and Practice. Signal Processing. 
Bygrave, A.M., Jahans-Price, T., Wolff, A.R., Sprengel, R., Kullmann, D.M., Bannerman, D.M., and Katzel, D. (2019). Hippocampal-prefrontal coherence mediates working memory and selective attention at distinct frequency bands and provides a causal link between schizophrenia and its risk gene GRIA1. Transl Psychiatry 9, 142.

Cane, M., Maco, B., Knott, G., and Holtmaat, A. (2014). The relationship between PSD-95 clustering and spine stability in vivo. J Neurosci 34, 2075-2086.

Chen, H., Roth, R.H., Lopez-Ortega, E., Tan, H.L., and Huganir, R.L. (2021). AMPA Receptors Exist in Tunable Mobile and Immobile Synaptic Fractions In Vivo. eNeuro 8.

Diaz-Alonso, J., and Nicoll, R.A. (2021). AMPA receptor trafficking and LTP: Carboxy-termini, aminotermini and TARPs. Neuropharmacology 197, 108710.

Diaz-Alonso, J., Sun, Y.J., Granger, A.J., Levy, J.M., Blankenship, S.M., and Nicoll, R.A. (2017). Subunitspecific role for the amino-terminal domain of AMPA receptors in synaptic targeting. Proc Natl Acad Sci U S A 114, 7136-7141.

Diering, G.H., Nirujogi, R.S., Roth, R.H., Worley, P.F., Pandey, A., and Huganir, R.L. (2017). Homer1a drives homeostatic scaling-down of excitatory synapses during sleep. Science 355, 511-515.

Dombeck, D.A., Harvey, C.D., Tian, L., Looger, L.L., and Tank, D.W. (2010). Functional imaging of hippocampal place cells at cellular resolution during virtual navigation. Nat Neurosci 13, 1433-1440.

El-Boustani, S., Ip, J.P.K., Breton-Provencher, V., Knott, G.W., Okuno, H., Bito, H., and Sur, M. (2018). Locally coordinated synaptic plasticity of visual cortex neurons in vivo. Science 360, 1349-1354.

Epsztein, J., Brecht, M., and Lee, A.K. (2011). Intracellular determinants of hippocampal CA1 place and silent cell activity in a novel environment. Neuron 70, 109-120.

Falk, T., Mai, D., Bensch, R., Cicek, O., Abdulkadir, A., Marrakchi, Y., Bohm, A., Deubner, J., Jackel, Z., Seiwald, K., et al. (2019). U-Net: deep learning for cell counting, detection, and morphometry. Nat Methods 16, 67-70.

Fang, H., Bygrave, A.M., Roth, R.H., Johnson, R.C., and Huganir, R.L. (2021). An optimized CRISPR/Cas9 approach for precise genome editing in neurons. Elife 10.

Fenno, L., Yizhar, O., and Deisseroth, K. (2011). The development and application of optogenetics. Annu Rev Neurosci 34, 389-412.

Fortin, D.A., Tillo, S.E., Yang, G., Rah, J.C., Melander, J.B., Bai, S., Soler-Cedeno, O., Qin, M., Zemelman, B.V., Guo, C., et al. (2014). Live imaging of endogenous PSD-95 using ENABLED: a conditional strategy to fluorescently label endogenous proteins. J Neurosci 34, 16698-16712.

Frey, U., and Morris, R.G. (1997). Synaptic tagging and long-term potentiation. Nature 385, 533-536.

Frischknecht, R., Heine, M., Perrais, D., Seidenbecher, C.I., Choquet, D., and Gundelfinger, E.D. (2009). Brain extracellular matrix affects AMPA receptor lateral mobility and short-term synaptic plasticity. Nat Neurosci 12, 897-904.

Gamache, T.R., Araki, Y., and Huganir, R.L. (2020). Twenty Years of SynGAP Research: From Synapses to Cognition. J Neurosci 40, 1596-1605. 
Gambino, F., Pages, S., Kehayas, V., Baptista, D., Tatti, R., Carleton, A., and Holtmaat, A. (2014). Sensory-evoked LTP driven by dendritic plateau potentials in vivo. Nature 515, 116-119.

Gray, N.W., Weimer, R.M., Bureau, I., and Svoboda, K. (2006). Rapid redistribution of synaptic PSD-95 in the neocortex in vivo. PLoS Biol 4, e370.

Henley, J.M., and Wilkinson, K.A. (2016). Synaptic AMPA receptor composition in development, plasticity and disease. Nat Rev Neurosci 17, 337-350.

Hochbaum, D.a.S., DB (1985). A Best Possible Heuristic for the k-Center Problem. Mathematics of Operations Research 10, 175-366.

Holtmaat, A., Bonhoeffer, T., Chow, D.K., Chuckowree, J., De Paola, V., Hofer, S.B., Hubener, M., Keck, T., Knott, G., Lee, W.C., et al. (2009). Long-term, high-resolution imaging in the mouse neocortex through a chronic cranial window. Nat Protoc 4, 1128-1144.

Holtmaat, A., and Caroni, P. (2016). Functional and structural underpinnings of neuronal assembly formation in learning. Nat Neurosci 19, 1553-1562.

Holtmaat, A., and Svoboda, K. (2009). Experience-dependent structural synaptic plasticity in the mammalian brain. Nat Rev Neurosci 10, 647-658.

Holtmaat, A., Wilbrecht, L., Knott, G.W., Welker, E., and Svoboda, K. (2006). Experience-dependent and cell-type-specific spine growth in the neocortex. Nature 441, 979-983.

Huganir, R.L., and Nicoll, R.A. (2013). AMPARs and synaptic plasticity: the last 25 years. Neuron 80 , 704-717.

Juavinett, A.L., Bekheet, G., and Churchland, A.K. (2019). Chronically implanted Neuropixels probes enable high-yield recordings in freely moving mice. Elife 8.

Jun, J.J., Steinmetz, N.A., Siegle, J.H., Denman, D.J., Bauza, M., Barbarits, B., Lee, A.K., Anastassiou, C.A., Andrei, A., Aydin, C., et al. (2017). Fully integrated silicon probes for high-density recording of neural activity. Nature 551, 232-236.

Kopec, C.D., Li, B., Wei, W., Boehm, J., and Malinow, R. (2006). Glutamate receptor exocytosis and spine enlargement during chemically induced long-term potentiation. J Neurosci 26, 2000-2009.

Lee, K.F., Soares, C., and Beique, J.C. (2012). Examining form and function of dendritic spines. Neural Plast 2012, 704103.

Lee S.H., Jin C., Cai E., Ge P., Ishitsuka Y., Teng K.W., de Thomaz A.A., Nall D., Baday M., Jeyifous O., Demonte D., Dundas C.M., Park S., Delgado J.Y., Green W.N., Selvin P.R. (2017). Super-resolution imaging of synaptic and Extra-synaptic AMPA receptors with different-sized fluorescent probes. eLife 6:e27744. doi: 10.7554/eLife.27744.

Li, T.P., Song, Y., MacGillavry, H.D., Blanpied, T.A., and Raghavachari, S. (2016). Protein Crowding within the Postsynaptic Density Can Impede the Escape of Membrane Proteins. J Neurosci 36, 42764295.

Lin, M.Z., and Schnitzer, M.J. (2016). Genetically encoded indicators of neuronal activity. Nat Neurosci $19,1142-1153$. 
Makino, H., and Malinow, R. (2009). AMPA receptor incorporation into synapses during LTP: the role of lateral movement and exocytosis. Neuron 64, 381-390.

Malinow, R., and Malenka, R.C. (2002). AMPA receptor trafficking and synaptic plasticity. Annu Rev Neurosci 25, 103-126.

Mammen, A.L., Huganir, R.L., O'Brien, R.J. (1997). Redistribution and stabilization of cell surface glutamate receptors during synapse formation. J. Neurosci 17, 7351-7358

Martin, S., Henley, J.M., Holman, D., Zhou, M., Wiegert, O., van Spronsen, M., Joels, M., Hoogenraad, C.C., and Krugers, H.J. (2009). Corticosterone alters AMPAR mobility and facilitates bidirectional synaptic plasticity. PLoS One 4, e4714.

Matsuzaki, M., Ellis-Davies, G.C., Nemoto, T., Miyashita, Y., lino, M., and Kasai, H. (2001). Dendritic spine geometry is critical for AMPA receptor expression in hippocampal CA1 pyramidal neurons. Nat Neurosci 4, 1086-1092.

McNaughton, B.L., Douglas, R.M., and Goddard, G.V. (1978). Synaptic enhancement in fascia dentata: cooperativity among coactive afferents. Brain Res 157, 277-293.

Miesenbock, G., De Angelis, D.A., and Rothman, J.E. (1998). Visualizing secretion and synaptic transmission with pH-sensitive green fluorescent proteins. Nature 394, 192-195.

Milletari, F., Navab, N, and Seyed-Ahmad Ahmadi (2016). V-net: Fully convolutional neural networks for volumetric medical image segmentation. IEEE.

Miyamoto, D., Marshall, W., Tononi, G., and Cirelli, C. (2021). Net decrease in spine-surface GluA1containing AMPA receptors after post-learning sleep in the adult mouse cortex. Nat Commun 12, 2881.

Moen, E., Bannon, D., Kudo, T., Graf, W., Covert, M., and Van Valen, D. (2019). Deep learning for cellular image analysis. Nat Methods 16, 1233-1246.

Nabavi, S., Fox, R., Alfonso, S., Aow, J., and Malinow, R. (2014). GluA1 trafficking and metabotropic NMDA: addressing results from other laboratories inconsistent with ours. Philos Trans $R$ Soc Lond B Biol Sci 369, 20130145.

Nicoll, R.A. (2017). A Brief History of Long-Term Potentiation. Neuron 93, 281-290.

O'Brien, R.J., Kamboj, S., Ehlers, M.D., Rosen, K.R., Fischbach, G.D., and Huganir, R.L. (1998). Activitydependent modulation of synaptic AMPA receptor accumulation. Neuron 21, 1067-1078.

Olaf Ronneberger, P.F., and Thomas Brox (2015). U-net: Convolutional networks for biomedical image segmentation. International Conference on Medical image computing and computer-assisted intervention.

Patterson, M.A., Szatmari, E.M., and Yasuda, R. (2010). AMPA receptors are exocytosed in stimulated spines and adjacent dendrites in a Ras-ERK-dependent manner during long-term potentiation. Proc Natl Acad Sci U S A 107, 15951-15956.

Rosenkrantz, D.S.R.L.P. (1977). An Analysis of Several Heuristics for the Traveling Salesman Problem. SIAM Journal on Computing 6, 563-581. 
Roth, R.H., Cudmore, R.H., Tan, H.L., Hong, I., Zhang, Y., and Huganir, R.L. (2019). Cortical Synaptic AMPA Receptor Plasticity during Motor Learning. Neuron.

Roth, R.H., Zhang, Y., and Huganir, R.L. (2017). Dynamic imaging of AMPA receptor trafficking in vitro and in vivo. Curr Opin Neurobiol 45, 51-58.

Sanderson, D.J., Gray, A., Simon, A., Taylor, A.M., Deacon, R.M., Seeburg, P.H., Sprengel, R., Good, M.A., Rawlins, J.N., and Bannerman, D.M. (2007). Deletion of glutamate receptor-A (GluR-A) AMPA receptor subunits impairs one-trial spatial memory. Behav Neurosci 121, 559-569.

Santuy, A., Tomas-Roca, L., Rodriguez, J.R., Gonzalez-Soriano, J., Zhu, F., Qiu, Z., Grant, S.G.N., DeFelipe, J., and Merchan-Perez, A. (2020). Estimation of the number of synapses in the hippocampus and brain-wide by volume electron microscopy and genetic labeling. Sci Rep 10, 14014.

Schindelin, J., Arganda-Carreras, I., Frise, E., Kaynig, V., Longair, M., Pietzsch, T., Preibisch, S., Rueden, C., Saalfeld, S., Schmid, B., et al. (2012). Fiji: an open-source platform for biological-image analysis. Nat Methods 9, 676-682.

Sdrulla, A.D., and Linden, D.J. (2007). Double dissociation between long-term depression and dendritic spine morphology in cerebellar Purkinje cells. Nat Neurosci $10,546-548$.

Sharma, K., Fong, D.K., and Craig, A.M. (2006). Postsynaptic protein mobility in dendritic spines: longterm regulation by synaptic NMDA receptor activation. Mol Cell Neurosci 31, 702-712.

Sheng, M., Sabatini, B.L., and Sudhof, T.C. (2012). Synapses and Alzheimer's disease. Cold Spring Harb Perspect Biol 4.

Sheng, N., Bemben, M.A., Diaz-Alonso, J., Tao, W., Shi, Y.S., and Nicoll, R.A. (2018). LTP requires postsynaptic PDZ-domain interactions with glutamate receptor/auxiliary protein complexes. Proc Natl Acad Sci U S A 115, 3948-3953.

Sofroniew, N.J., Flickinger, D., King, J., and Svoboda, K. (2016). A large field of view two-photon mesoscope with subcellular resolution for in vivo imaging. Elife 5.

Spruston, N. (2008). Pyramidal neurons: dendritic structure and synaptic integration. Nat Rev Neurosci 9, 206-221.

Suresh, A., and Dunaevsky, A. (2017). Relationship Between Synaptic AMPAR and Spine Dynamics: Impairments in the FXS Mouse. Cereb Cortex 27, 4244-4256.

Tan, H.L., Roth, R.H., Graves, A.R., Cudmore, R.H., and Huganir, R.L. (2020). Lamina-specific AMPA receptor dynamics following visual deprivation in vivo. Elife 9.

Trachtenberg, J.T., Chen, B.E., Knott, G.W., Feng, G., Sanes, J.R., Welker, E., and Svoboda, K. (2002). Long-term in vivo imaging of experience-dependent synaptic plasticity in adult cortex. Nature 420, 788794.

Turrigiano, G.G., Leslie, K.R., Desai, N.S., Rutherford, L.C., and Nelson, S.B. (1998). Activity-dependent scaling of quantal amplitude in neocortical neurons. Nature 391, 892-896.

Tward, D., Brown, T., Kageyama, Y., Patel, J., Hou, Z., Mori, S., Albert, M., Troncoso, J., and Miller, M. (2020). Diffeomorphic Registration With Intensity Transformation and Missing Data: Application to 3D Digital Pathology of Alzheimer's Disease. Front Neurosci 14, 52. 
Volk, L., Chiu, S.L., Sharma, K., and Huganir, R.L. (2015). Glutamate synapses in human cognitive disorders. Annu Rev Neurosci 38, 127-149.

Xu, N.L., Harnett, M.T., Williams, S.R., Huber, D., O'Connor, D.H., Svoboda, K., and Magee, J.C. (2012). Nonlinear dendritic integration of sensory and motor input during an active sensing task. Nature 492, 247-251.

Xu, T., Yu, X., Perlik, A.J., Tobin, W.F., Zweig, J.A., Tennant, K., Jones, T., and Zuo, Y. (2009). Rapid formation and selective stabilization of synapses for enduring motor memories. Nature 462, 915-919.

Yang, G., Pan, F., and Gan, W.B. (2009). Stably maintained dendritic spines are associated with lifelong memories. Nature 462, 920-924.

Zamanillo, D., Sprengel, R., Hvalby, O., Jensen, V., Burnashev, N., Rozov, A., Kaiser, K.M., Koster, H.J., Borchardt, T., Worley, P., et al. (1999). Importance of AMPA receptors for hippocampal synaptic plasticity but not for spatial learning. Science 284, 1805-1811.

Zhang, Y., Cudmore, R.H., Lin, D.T., Linden, D.J., and Huganir, R.L. (2015). Visualization of NMDA receptor-dependent AMPA receptor synaptic plasticity in vivo. Nat Neurosci 18, 402-407.

Zhou, Z., Liu, A., Xia, S., Leung, C., Qi, J., Meng, Y., Xie, W., Park, P., Collingridge, G.L., and Jia, Z. (2018). The C-terminal tails of endogenous GluA1 and GluA2 differentially contribute to hippocampal synaptic plasticity and learning. Nat Neurosci 21, 50-62.

Zhu, F., Cizeron, M., Qiu, Z., Benavides-Piccione, R., Kopanitsa, M.V., Skene, N.G., Koniaris, B., DeFelipe, J., Fransen, E., Komiyama, N.H., et al. (2018). Architecture of the Mouse Brain Synaptome. Neuron 99, 781-799 e710. 